\title{
REPRESENTATION THEORY OF SOME INFINITE-DIMENSIONAL ALGEBRAS ARISING IN CONTINUOUSLY CONTROLLED ALGEBRA AND TOPOLOGY
}

\author{
FERNANDO MURO
}

\section{Contents}

1. Introduction

1.1. Notation and conventions 3

2. Ringoids and modules 4

3. The algebras $R(E)$ and related additive categories 5

3.1. The special case card $E$ finite $\quad 7$

4. Countably presented $R$-modules as finitely presented $\operatorname{RCFM}(R)$-modules 8

5. A review on pro-categories $\quad 10$

6. Numerical invariants of finitely presented $k(\boldsymbol{n})$-modules 10

7. Classification of finitely presented $\operatorname{RCFM}(k)$-modules 12

8. Representations of the $n$-subspace quiver 22

9. Finitely presented $k(\mathbf{n})$-modules and finite-dimensional $n$-subspaces 24

10. Classification of finitely presented $k(\mathbf{n})$-modules $\quad 29$

Appendix A. Some computations of $\operatorname{Ext}_{k(\boldsymbol{n})}^{1}$ groups 30

References $\quad 32$

\section{INTRODUCTION}

Suppose that the discrete set $\mathbb{N}_{0}$ of non-negative integers is embedded $\mathbb{N}_{0} \subset X$ in a compact metrizable space $X$, and let $E=\mathbb{N}_{0}^{\prime} \subset X$ be the derived set (i. e. points of $X$ which contain infinitely many points of $\mathbb{N}_{0}$ in any neighborhood). Consider the set $R(E)$ of $\mathbb{N}_{0} \times \mathbb{N}_{0}$ matrices $\left(\mathrm{a}_{i j}\right)_{i, j \in \mathbb{N}_{0}}$ with entries in a (unital associative) ring $R$ such that if $\left\{i_{n}\right\}_{n \in \mathbb{N}_{0}},\left\{j_{n}\right\}_{n \in \mathbb{N}_{0}} \subset \mathbb{N}_{0}$ are sequences convergent in $X$ to different points then the vector $\left(a_{i_{n} j_{n}}\right)_{n \in \mathbb{N}_{0}}$ is almost all zero. This set is an $R$-algebra with the usual matrix operations. Any compact metrizable space can arise as $E$ in this way. In fact the isomorphism class of the algebra $R(E)$ only depends on $E$. These algebras are Morita equivalent to some additive categories of free $R$-modules continuously controlled at infinity by $E$ appearing in the literature. These categories play an important role in many areas such as controlled homotopy theory, proper homotopy theory, $C^{*}$-algebra theory, $K$-theory and $L$-theory, see for example [15], [5], [9], [2] and [1].

The elementary properties of the algebras $R(E)$ have been studied by BauesQuintero ([2]) for $R=\mathbb{Z}$ the integers. If $E$ is zero-dimensional, $R(E)$ is a particular case of the rings considered by Farrell-Wagoner ([7]). When $E=*$ is a singleton $R(E)=\operatorname{RCFM}(R)$ is the well-known algebra of row-column-finite (or locally finite) 
matrices over $R$. This algebra has been studied from a purely ring-theoretical point of view, see for example [17]. It was also used by Wagoner ([18]) to construct deloopings in algebraic $K$-theory.

In this paper we concentrate on the representation theory of the algebras $k(E)$, where $k$ is any field.

Representation theory considers the decomposition problem in a small additive category A. A solution to this problem consists of a set of objects (which we call elementary objects) and of a set of isomorphisms (elementary isomorphisms) between finite direct sums of elementary objects. These sets must satisfy the following properties: any object in $\mathbf{A}$ is isomorphic to a finite direct sum of elementary ones, and any isomorphism relation between two such direct sums can be derived from the elementary isomorphisms. Notice that this is exactly a presentation of the abelian monoid Iso(A) of isomorphisms classes of objects in $\mathbf{A}$. The trivial solution is taking all objects as elementary objects and all isomorphisms as elementary isomorphisms, however one is often interested in solutions minimizing the cardinal of the sets of elementary objects and isomorphisms.

We say that $\mathbf{A}$ has finite representation type if there exists a finite set of elementary objects, or equivalently $\operatorname{Iso}(\mathbf{A})$ is finitely presented. The representation type of $\mathbf{A}$ is wild if a solution to the decomposition problem in $\mathbf{A}$ would yield a solution to the decomposition problem in the category of finite-dimensional modules over a polynomial $k$-algebra in two non-commuting variables. Otherwise $\mathbf{A}$ has tame representation type. If $\mathbf{A}$ has wild representation type the word problem for finitely presented groups, which is undecidable, can be embedded in the decomposition problem in $\mathbf{A}$, hence one can not expect to get satisfactory solutions in this case. The representation type of an algebra $A$ is that of the category $\mathbf{f p}(A)$ of finitely presented (right) $A$-modules.

One of the main results of this paper is the following theorem, where we compute the representation type of the algebra $k(E)$ in terms of the cardinal of $E$, without restrictions on the ground field $k$.

Theorem 1.1. The representation type of $k(E)$ is

\begin{tabular}{|c|c|}
\hline card $E$ & type \\
\hline$<4$ & finite \\
$=4$ & tame \\
$>4$ & wild \\
\hline
\end{tabular}

In the finite and tame cases we construct explicit presentations of $\operatorname{Iso}(\mathbf{f p}(k(E)))$. Moreover, for $E$ finite, we prove that there are presentations of $\operatorname{Iso}(\mathbf{f p}(k(E)))$ with a finite number of elementary isomorphisms and we compute them. These presentations satisfy the next properties.

Theorem 1.2. If card $E$ is finite there are solutions to the decomposition problem in $\mathbf{f} \mathbf{p}(k(E))$ with the next cardinals of elementary modules and isomorphisms

\begin{tabular}{|c|c|c|}
\hline card $E$ & modules & isomorphisms \\
\hline 1 & 6 & 6 \\
2 & 12 & 12 \\
3 & 21 & 18 \\
$\geq 4$ & $\geq \aleph_{0}$ & 6 card $E$ \\
\hline
\end{tabular}


There are two key steps in the proof of these results. The first is to solve the decomposition problem for finitely presented $\operatorname{RCFM}(k)$-modules. The second is to relate the decomposition problem in $\mathbf{f p}(k(E))$ when $\operatorname{card} E=n$ is finite to the decomposition problems in $\mathbf{f p}(\operatorname{RCFM}(k))$ and in the category of finite-dimensional $n$-subspaces.

We shall use the pro-category of pro-vector spaces and the inverse limit functor to construct invariants detecting isomorphism types of finitely presented $k(E)$ modules. Moreover, we shall not usually work directly with the categories of finitely presented $k(E)$-modules but with the equivalent categories of finitely presented modules over certain small additive categories. This setting allows more flexibility and technical proofs become less complicated than if we use $k(E)$-modules.

In order to facilitate the reading we now describe the contents of this paper. In the next section we briefly recall from [12] the basic tools of ringoid theory that we need. Afterwards, in Section 3, we introduce the ringoids which are Morita equivalent to the algebras $R(E)$ and establish their basic properties. For this we use the approach in [2], generalizing some results in this reference for $R=\mathbb{Z}$ to arbitrary rings. We put emphasis on the case $E$ finite because we shall always work under this assumption (even for the proof of Theorem 1.1, see Remark 3.10). In Section 4 we construct an embedding of the decomposition problem for countably presented $R$ modules into the decomposition problem for finitely presented $R(E)$-modules, where $E$ is any non-empty compact metrizable space. Section 5 contains basic facts about pro-categories. In Section 6 we construct some invariants of the isomorphism class of a finitely presented $k(E)$-module, $E$ finite. These invariants are used in Section 7 to classify finitely presented $k(E)$-modules when $E=*$ is just one point and $k(E)=$ $\operatorname{RCFM}(k)$. In particular we prove that this $k$-algebra has finite representation type. The classification theorem (Theorem 7.1) is derived from several technical lemmas. In Section 8 we recall the definition and representation theory of the $n$-subspace quiver. We also define the class of rigid $n$-subspaces, which plays an important role in what follows. We show that all but $3 n$ indecomposable representations of the $n$-subspace quiver are rigid $n$-subspaces. In Section 9 we relate the representation theories of both $k(E)$ and the $n$-subspace quiver, where $n$ is the cardinal of $E$. The properties of this relation are established through many technical results which lead us to complete the proofs of Theorems 1.1 and 1.2 in Section 10. In this last section we compute the structure of the monoid $\operatorname{Iso}(\mathbf{f p}(k(E)))$ (Theorem 10.1) and give a classification theorem for finitely presented $k(E)$-modules (Corollary $10.5)$ for $E$ finite. This classification theorem explicitly describes the (finite) set of elementary isomorphisms, and also the set of elementary objects when $E$ has less than 5 points. We include an Appendix with some computations of $\operatorname{Ext}^{1}$ groups of finitely presented $k(E)$-modules, $E$ finite. These computations will be applied to proper homotopy theory in a forthcoming paper ([13]).

1.1. Notation and conventions. In this paper all rings and algebras are associative with unit. We use bold letters $\mathbf{C}$ for categories, $R$ for an arbitrary (non-commutative) ring, $\mathbb{Z}$ for the ring of integers, and $k$ for fields. As usual $\mathbb{N}=\{1,2,3, \ldots\}$ is the set of natural numbers, and $\mathbb{N}_{0}=\mathbb{N} \cup\{0\}$ the free abelian monoid with one generator.

Capital sans serif letters $A$ are names of matrices with entries in some ring. Here all matrices are square matrices indexed by $\mathbb{N}_{0}$, and the entry of a matrix $A$ corresponding to the subindexes $i, j \in \mathbb{N}_{0}$ is denoted by $\mathrm{a}_{i j}$, it is $\mathrm{A}=\left(\mathrm{a}_{i j}\right)_{i, j \in \mathbb{N}_{0}}$. 
The identity matrix is denoted by $\mathbf{I}$, it is defined as $\mathbf{i}_{i i}=1\left(i \in \mathbb{N}_{0}\right)$ and $\mathbf{i}_{i j}=0$ for $i \neq j$. The entries of the transposed matrix $\mathrm{A}^{t}=\left(\mathrm{a}_{i j}^{t}\right)_{i, j \in \mathbb{N}_{0}}$ of $\mathrm{A}$ are $\mathrm{a}_{i j}^{t}=\mathrm{a}_{j i}$ $\left(i, j \in \mathbb{N}_{0}\right)$. Vectors are denoted by $\left(v_{i}\right)_{i=1}^{n}$ or $\left(v_{n}\right)_{n \in \mathbb{N}_{0}}$ provided they have a finite or an infinite countable number of entries. We regard vectors as column matrices, hence matrices act on vectors on the left.

\section{RingoidS AND MODULES}

A ringoid $\mathbf{R}$ is a category whose morphism sets $\operatorname{Hom}_{\mathbf{R}}(X, Y)$ are abelian groups in such a way that composition is bilinear. The endomorphism set $\operatorname{End}_{\mathbf{R}}(X)=$ $\operatorname{Hom}_{\mathbf{R}}(X, X)$ of an object $X$ has a ring structure with product given by composition of morphisms. Conversely any ring $R$ is identified with the ringoid with a single object whose endomorphism set is $R$. An additive category is a ringoid with finite biproducts (direct sums). In this section we recall basic facts about modules over a small ringoid. Our main reference for this subject is [12].

An additive functor between ringoids is a functor which induces homomorphisms between morphism sets. Let $\mathbf{A b}$ be the category of abelian groups. A right-R-module $\mathcal{M}$ is an additive functor $\mathcal{M}: \mathbf{R}^{o p} \rightarrow \mathbf{A b}$. Morphisms of right$\mathbf{R}$-modules are natural transformations, and the category of right-R-modules is denoted by $\bmod (\mathbf{R})$ whenever $\mathbf{R}$ is small. Left-R-modules are the same thing as right- $\mathbf{R}^{o p}$-modules, where $\mathbf{R}^{o p}$ is the opposite category, so every statement about right-modules has a convenient translation to left-modules. From now on every module is a right-module unless we state the contrary.

There is a Yoneda full inclusion of categories $\mathbf{R} \subset \bmod (\mathbf{R})$ which sends an object $X$ in $\mathbf{R}$ to the associated contravariant representable functor $\operatorname{Hom}_{\mathbf{R}}(-, X)$. These $\mathbf{R}$-modules are said to be finitely generated free. They are projective by Yoneda's lemma.

An $\mathbf{R}$-module $\mathcal{M}$ is finitely presented (f. p.) if it is the cokernel of a morphism between two finite direct sums of finitely generated free $\mathbf{R}$-modules. The cokernel of a morphism between $\mathrm{f}$. p. modules is also f. p. In particular direct summands of $\mathrm{f}$. p. modules are f. p. We write $\mathbf{f} p(\mathbf{R}) \subset \bmod (\mathbf{R})$ for the full subcategory of f. p. $\mathbf{R}$ modules. If $\mathbf{R}=\mathbf{A}$ is an additive category, then a $\mathrm{f}$. $\mathrm{p}$. A-module $\mathcal{M}$ is in fact the cokernel in $\bmod (\mathbf{A})$ of a morphism $\varphi: X_{1} \rightarrow X_{0}$ in $\mathbf{A}$. One can readily check that if $\mathcal{N}=\operatorname{Coker}\left[\psi: Y_{1} \rightarrow Y_{0}\right]$ is another f. p. A-module, any morphism $\tau: \mathcal{M} \rightarrow \mathcal{N}$ is represented by a morphism $\tau_{0}: X_{0} \rightarrow Y_{0}$ such that there exists $\tau_{1}: X_{1} \rightarrow Y_{1}$ with $\tau_{0} \varphi=\psi \tau_{1}$. Another morphism $\tau_{0}^{\prime}:: X_{0} \rightarrow Y_{0}$ represents $\tau$ if and only if there exists $\eta: X_{0} \rightarrow Y_{1}$ with $\tau_{0}+\psi \eta=\tau_{0}^{\prime}$. More precisely, let pair(A) be the additive category whose objects are morphisms in $\mathbf{A}$, and morphisms $\tau=\left(\tau_{1}, \tau_{0}\right): \varphi \rightarrow \psi$ are commutative squares

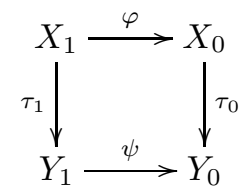

There is an obvious functor Coker: pair $(A) \rightarrow \mathbf{f p}(\mathbf{A})$ given by taking cokernels. We define in $\operatorname{pair}(\mathbf{A})$ the natural equivalence relation $\sim$ with $\tau \sim \tau^{\prime}$ if there exists $\eta: X_{0} \rightarrow Y_{1}$ satisfying $\tau_{0}+\psi \eta=\tau_{0}^{\prime}$.

Proposition 2.1. The functor Coker factors through the quotient category $\operatorname{pair}(\mathbf{A}) / \sim$ and the induced functor Coker: $\operatorname{pair}(\mathbf{A}) / \sim \rightarrow \mathbf{f p}(\mathbf{A})$ is an equivalence of categories. 
Any additive functor $\mathbb{F}: \mathbf{R} \rightarrow \mathbf{S}$ between small ringoids induces two "change of coefficients" additive functors $\mathbb{F}^{*}: \bmod (\mathbf{S}) \rightarrow \bmod (\mathbf{R})$ and $\mathbb{F}_{*}: \bmod (\mathbf{R}) \rightarrow$ $\bmod (\mathbf{S})$. The first one is exact and sends an $\mathbf{S}$-module $\mathcal{M}$ to the composite $\mathbb{F}^{*} \mathcal{M}=$ $\mathcal{M F}$. The second one is left-adjoint to $\mathbb{F}^{*}\left(\mathbb{F}_{*}\right.$ is the left additive Kan extension along $\mathbb{F}$, see [12] 6) and hence right-exact. Moreover, the next diagram commutes

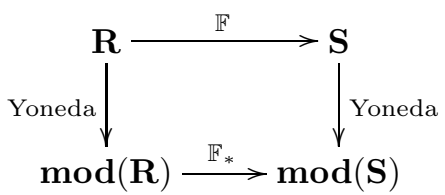

In addition if $\mathbb{F}$ is full and faithful then so is $\mathbb{F}_{*}$, and in this case $\mathbb{F}^{*} \mathbb{F}_{*}$ is naturally equivalent to the identity, see [3] 3.4.1. This follows from the fact that any $\mathbf{R}$-module admits a projective resolution by (arbitrary) direct sums of finitely generated free ones. The functor $\mathbb{F}_{*}$ restricts to the full subcategories of $\mathrm{f}$. p. modules.

If we identify the endomorphism $\operatorname{ring} \operatorname{End}_{\mathbf{R}}(X)$ of an object $X$ with the full subcategory of $\mathbf{R}$ whose unique object is $X$ the change of coefficients $\mathbb{F}^{*}$ induced by the inclusion $\mathbb{F}: \operatorname{End}_{\mathbf{R}}(X) \subset \mathbf{R}$ is the evaluation functor

$$
e v_{X}=\mathbb{F}^{*}: \bmod (\mathbf{R}) \longrightarrow \bmod \left(\operatorname{End}_{\mathbf{R}}(X)\right): \mathcal{M} \mapsto \mathcal{M}(X) \text {. }
$$

The next proposition is an useful criterion to detect when a ringoid is Morita equivalent to a ring. It is a consequence of [12] 8.1.

Proposition 2.2. If every object in $\mathbf{R}$ is a retract of $X$ then the evaluation functor $e v_{X}$ is an additive equivalence of abelian categories which restricts to an equivalence between the full subcategories of finitely presented modules.

In a more categorical language ringoids are defined as categories enriched over the monoidal category of abelian groups with the usual tensor product, compare [4] 6.2. For any ring $R$ one can consider the monoidal category of $R$ - $R$-bimodules with the $R$-tensor product and define an $R$-ringoid as a category enriched over it. This is the same as an $R$-category in the sense of [12] when $R$ is commutative. If $\mathbf{R}$ is an $R$-ringoid the endomorphism ring $\operatorname{End}_{\mathbf{R}}(X)$ of an object $X$ is in fact an $R$-algebra. In this case $\mathbf{R}$-modules and morphisms between them take values in the category of (right) $R$-modules in a natural way.

\section{The algebras $R(E)$ AND Related AdDitive CAtegories}

Given a ring $R$ and a set $A$ we write $R\langle A\rangle$ for the free $R$-module with basis set $A$. Free $R$-modules are $R$ - $R$-bimodules, hence the additive category of free $R$-modules and right- $R$-module homomorphisms is an $R$-ringoid. The carrier of an element $x \in R\langle A\rangle$ is the (finite) set $\operatorname{carr}(x) \subset A$ such that $z \in \operatorname{carr}(x)$ if $z$ appears with a non-trivial coefficient in the linear expansion of $x$.

For every non-empty compact metrizable space $E$ there exists another one $X$ containing $E$ such that the complement $Y=X-E$ is dense in $X$. The triple $\bar{T}=(X, Y, E)$ can always be chosen to be a tree-like space in the sense of [2] III.1.1. But one can also take $X$ to be the (unreduced) cone over $E, X=C E=$ $E \times[0,1] / E \times\{1\}$. Here we identify $E$ with $E \times\{0\}$ inside the cone $C E$.

A free $\bar{T}$-controlled $R$-module $R\langle A\rangle_{\alpha}$ is a free $R$-module $R\langle A\rangle$ together with a function $\alpha: A \rightarrow Y$, called height function, such that $\alpha^{-1}(K)$ is finite for every compact subspace $K \subset Y$. The set $A$ is necessarily countable and the derived 
set of $\alpha(A)$ in $X$ satisfies $\alpha(A)^{\prime} \subset E$. This derived set is the support of $R\langle A\rangle_{\alpha}$. Controlled homomorphisms $\varphi: R\langle A\rangle_{\alpha} \rightarrow R\langle B\rangle_{\beta}$ are homomorphisms between the underlying $R$-modules such that for every $x \in E$ and every neighborhood $U$ of $x$ in $X$ there exists another neighborhood $V \subset U$ of $x$ in $X$ such that if $a \in A$ satisfies $\alpha(a) \in V$ then $\beta(\operatorname{carr}(\varphi(a))) \subset U$. The category $\mathbf{M}_{R}(\bar{T})$ of free $\bar{T}$-controlled $R$ modules and controlled homomorphisms is a small additive category. Moreover, it is an $R$-ringoid. The sum and $R$-actions on morphism sets are given by those of the underlying free $R$-module homomorphisms, and the direct sum of two objects is $R\langle A\rangle_{\alpha} \oplus R\langle B\rangle_{\beta}=R\langle A \sqcup B\rangle_{(\alpha, \beta)}$, where $A \sqcup B$ is the disjoin union of sets and $(\alpha, \beta): A \sqcup B \rightarrow Y$ is defined as $\alpha$ over $A$ and $\beta$ over $B$.

Remark 3.1. The category $\mathbf{M}_{R}(\bar{T})$ is defined in [2] III.4.7 for $\bar{T}$ a tree-like space. However, as it is pointed out in the Remark after that definition, it is equivalent to the category $\mathcal{B}(X, E ; R)$ in [5]. In particular $\mathbf{M}_{R}(\bar{T})$ only depends on $E$ up to equivalence of categories preserving supports of objects (in fact equivalence of $R$-ringoids), see 1.23 and 1.24 in [5].

The next proposition shows that free $\bar{T}$-controlled $R$-modules are classified by the underlying $R$-module and the support.

Proposition 3.2. Two free $\bar{T}$-controlled $R$-modules $R\langle A\rangle_{\alpha}, R\langle B\rangle_{\beta}$ are isomorphic if and only if the next two conditions are satisfied:

(1) The underlying $R$-modules are isomorphic $R\langle A\rangle \simeq R\langle B\rangle$,

(2) both have the same support $\alpha(A)^{\prime}=\beta(B)^{\prime}$.

If the supports are non-empty then condition (1) is automatically satisfied. Furthermore, any compact subset $K \subset E$ is the support of some free $\bar{T}$-controlled $R$-module.

In the proof of this proposition we shall use the following

Lemma 3.3. Given an injective controlled homomorphism $\varphi: R\langle A\rangle_{\alpha} \rightarrow R\langle B\rangle_{\beta}$ we have that $\alpha(A)^{\prime} \subset \beta(B)^{\prime}$.

Proof. For any $e \in \alpha(A)^{\prime}$ we can take a sequence $\left\{a_{n}\right\}_{n \in \mathbb{N}} \subset A$ with $\lim _{n \rightarrow \infty} \alpha\left(a_{n}\right)=$ $e$. Since $\varphi$ is injective $\operatorname{carr}\left(\varphi\left(a_{n}\right)\right)$ is non-empty for every $n \in \mathbb{N}$ so we can take elements $b_{n} \in \operatorname{carr}\left(\varphi\left(a_{n}\right)\right)$. By definition of controlled homomorphism $\lim _{n \rightarrow \infty} \beta\left(b_{n}\right)=$ $e$, hence $e \in \beta(B)^{\prime}$ and the inclusion holds.

Proof of (3.2). The case $R=\mathbb{Z}$ and $\bar{T}$ a tree-like space follows from [2] III.4.8 and III.4.16. In general condition (1) is necessary since an isomorphism of free $\bar{T}$-controlled $R$-modules is also an isomorphism between the underlying $R$-modules. Moreover, condition (2) is necessary by (3.3). By Remark 3.1 it is enough to make the proof for tree-like spaces. In the rest of the proof we shall suppose that $\bar{T}$ is tree-like.

If $\alpha(A)^{\prime}=\alpha(B)^{\prime}=\emptyset$ then $A$ and $B$ are both finite and any isomorphism $R\langle A\rangle \simeq$ $R\langle B\rangle$ is a controlled isomorphism $R\langle A\rangle_{\alpha} \simeq R\langle B\rangle_{\beta}$. If $\alpha(A)^{\prime}=\alpha(B)^{\prime} \neq \emptyset$ then $A$ and $B$ are infinite countable, so $\mathbb{Z}\langle A\rangle \simeq \mathbb{Z}\langle B\rangle$. Since the proposition holds for $R=\mathbb{Z}$ there is a controlled isomorphism $\varphi: \mathbb{Z}\langle A\rangle_{\alpha} \simeq \mathbb{Z}\langle B\rangle_{\beta}$. Now one can check that $\varphi \otimes R: R\langle A\rangle_{\alpha} \simeq R\langle B\rangle_{\beta}$ is an isomorphism of free $\bar{T}$-controlled $R$-modules.

Finally, given $K \subset E$ compact, if $\mathbb{Z}\langle C\rangle_{\gamma}$ is a free $\bar{T}$-controlled $\mathbb{Z}$-module whose support is $K$ then the support of $R\langle C\rangle_{\gamma}$ is $K$ as well, because it only depends on $\gamma$. The proof is now complete. 
The next result follows directly from Remark 3.1, Proposition 3.2 and the definition of controlled homomorphisms.

Proposition 3.4. Up to isomorphism, the endomorphism algebra of a free $\bar{T}$ controlled $R$-module with support $E$ only depends on $E$. Moreover, it is isomorphic to $R(E)$.

The last isomorphism of this proposition is given by the fact that the basis of a free $\bar{T}$-controlled $R$-module $R\langle A\rangle_{\alpha}$ with support $E$ must be infinite countable, and hence it can be identified with the non-negative integers $A=\mathbb{N}_{0}$. Moreover, we can suppose that $\alpha$ is the inclusion of a discrete subspace $\alpha: A \subset Y$, changing $A$ by $\alpha(A)$ if necessary. Now we are in the same situation as in the beginning of the introduction. We also derive from (3.4) that the isomorphism class of the $R$-algebra $R(E)$ only depends on $E$, as we claimed in the introduction.

Proposition 3.5. Every free $\bar{T}$-controlled $R$-module is a retract of any object whose support is $E$.

Proof. Recall from (3.2) that all objects with support $E$ are isomorphic. By (3.1) it is enough to check the proposition for $\bar{T}$ a tree-like space. For $R=\mathbb{Z}$ and $\bar{T}$ treelike this proposition is contained in the proof of [2] V.3.4. The result for arbitrary rings follows from the special case $R=\mathbb{Z}$. More precisely, given a free $\bar{T}$-controlled $R$-module $R\langle A\rangle_{\alpha}$ if the support of $R\langle B\rangle_{\beta}$ is $E$ then $\mathbb{Z}\langle A\rangle_{\alpha}$ is a retract of $\mathbb{Z}\langle B\rangle_{\beta}$ (the supports only depend on the height functions) hence we obtain a retraction of $R\langle B\rangle_{\beta}$ onto $R\langle A\rangle_{\alpha}$ by tensoring by $R$.

As a consequence of this proposition we get by (2.2) the following equivalence of categories which will be used from now on as an identification.

Corollary 3.6. The evaluation functor in a free $\bar{T}$-controlled $R$-module with support $E$ induces an additive equivalence of abelian categories $\bmod \left(\mathbf{M}_{R}(\bar{T})\right) \simeq \bmod (R(E))$ which restricts to another one $\mathbf{f} \mathbf{p}\left(\mathbf{M}_{R}(\bar{T})\right) \simeq \mathbf{f p}(R(E))$.

Remark 3.7. If $R \simeq R^{o p}$, in particular if $R$ is commutative, the transposition of matrices and an explicit isomorphism $R \simeq R^{o p}$ induce isomorphisms of $R$-ringoids $\mathbf{M}_{R}(\bar{T}) \simeq \mathbf{M}_{R}(\bar{T})^{o p}$ (preserving objects) and $R$-algebras $R(E) \simeq R(E)^{o p}$, compare [5], so in this case right-modules over $\mathbf{M}_{R}(\bar{T})$ or $R(E)$ are the same as left-modules.

In the following proposition we compute the dimension of the $k$-algebra $k(E), k$ any field.

Proposition 3.8. $\operatorname{dim} k(E)=2^{\aleph_{0}}$.

Proof. The $k$-vector space of all $\mathbb{N}_{0} \times \mathbb{N}_{0}$ matrices is the direct product of $\mathbb{N}_{0} \times \mathbb{N}_{0}$ copies of $k$, and it is known that $\operatorname{dim} \Pi_{\mathbb{N}_{0} \times \mathbb{N}_{0}} k=2^{\aleph_{0}}$, hence $\operatorname{dim} k(E) \leq 2^{\aleph_{0}}$. Moreover, for any element $\left(\mathrm{a}_{n}\right)_{n \in \mathbb{N}_{0}} \in \prod_{\mathbb{N}_{0}} k$ the diagonal matrix $\left(\mathrm{b}_{i j}\right)_{i, j \in \mathbb{N}_{0}}$ with $\mathrm{b}_{n n}=\mathrm{a}_{n}$ belongs to $k(E)$, therefore $k(E)$ has a vector subspace isomorphic to $\prod_{\mathbb{N}_{0}} k$, and $\operatorname{dim} \prod_{\mathbb{N}_{0}} k=2^{\aleph_{0}}$ as well, so the equality of the statement holds.

3.1. The special case card $E$ finite. If card $E$ is finite, since $E$ is metrizable, it must have the discrete topology, so $E$ is the discrete set $\boldsymbol{n}$ with $n=\operatorname{card} E$ points. For this particular space we can take a tree-like space $\bar{T}_{n}=\left(\hat{T}_{n}, T_{n}, \boldsymbol{n}\right)$ where $T_{n}$ is a locally compact tree with $n$ Freudenthal ends and $\hat{T}_{n}$ is the Freudenthal compactification of $T_{n}$, see [2] III.1.3. Moreover, if $T_{n}^{0}$ is the vertex set of $T_{n}$ and 
$\delta: T_{n}^{0} \subset T_{n}$ is the inclusion, the support of $R\left\langle T_{n}^{0}\right\rangle_{\delta}$ is $\mathbf{n}$, in particular $R(\mathbf{n})$ is the endomorphism ring of this object. Let us fix the following particular tree $T_{n}$ : the vertex set of $T_{n}$ is

$$
T_{n}^{0}=\left\{v_{0}\right\} \cup\left\{v_{m}^{1}, \ldots v_{m}^{n}\right\}_{m \geq 1},
$$

and there are edges joining $v_{0}$ with $v_{1}^{i}$ and $v_{m}^{i}$ with $v_{m+1}^{i}(1 \leq i \leq n, m \geq 1)$. The additive category $\mathbf{M}_{R}\left(\bar{T}_{n}\right)$ is equivalent to the full subcategory of objects $R\langle A\rangle_{\alpha}$ such that $\alpha(A) \subset T_{n}^{0}$, compare the proof of [2] V.3.4. From now on we shall always work in this subcategory, and we denote it by $\mathbf{M}_{R}\left(\bar{T}_{n}\right)$ as well.

We are going to give an alternative description for controlled homomorphisms in $\mathbf{M}_{R}\left(\bar{T}_{n}\right)$. For this we define the following sets for any height function $\alpha: A \rightarrow$ $T_{n}^{0} \subset T_{n}(1 \leq i \leq n, j \geq 1)$

$$
A_{j}^{i}=\bigcup_{l \geq j} \alpha^{-1}\left(v_{l}^{i}\right)
$$

A morphism $\varphi: R\langle B\rangle_{\beta} \rightarrow R\langle A\rangle_{\alpha}$ in $\mathbf{M}_{R}\left(\bar{T}_{n}\right)$ is controlled if and only if for every $m \geq 1$ there exists $M \geq 1$ such that $\varphi\left(B_{M}^{i}\right) \subset R\left\langle A_{m}^{i}\right\rangle$ for any $1 \leq i \leq n$. We shall omit the superindex $i$ when $n=1$. Moreover, for the next sections we fix the following notation $(m \geq 0)$

$$
{ }_{m} A=\alpha^{-1}\left(v_{0}\right) \cup\left[\bigcup_{1 \leq i \leq n} \bigcup_{l \leq m} \alpha^{-1}\left(v_{l}^{i}\right)\right],{ }_{m} A_{j}^{i}={ }_{m} A \cap A_{j}^{i} .
$$

Remark 3.9. If $n=1, T_{1}=[0,+\infty)$ is the half-line and $T_{1}^{0}=\mathbb{N}_{0}$ the non-negative integers. Moreover $R(\mathbf{1})$ is the $R$-algebra $\operatorname{RCFM}(R)$ of $\mathbb{N}_{0} \times \mathbb{N}_{0}$ matrices with entries in $R$ such that every row and every column has a finite number of non-zero entries (row-column-finite matrices), compare [2] V.3.8.

Remark 3.10. If $E$ is any compact metrizable space with at least $n$ points we can fully include $\mathbf{M}_{R}\left(\bar{T}_{n}\right)$ into $\mathbf{M}_{R}(\bar{T})$. For this we only need to take $n$ disjoint sequences $\left\{v_{m}^{i}\right\}_{m \geq 1}(1 \leq i \leq n)$ contained in $Y$ converging in $X$ to $n$ different points belonging to $E$, and an additional point $v_{0} \in Y$ out of the sequences. Now we identify $\mathbf{M}_{R}\left(\bar{T}_{n}\right)$ with the full subcategory of $\mathbf{M}_{R}(\bar{T})$ given by objects $R\langle A\rangle_{\alpha}$ with $\alpha(A) \subset\left\{v_{0}\right\} \cup\left\{v_{m}^{1}, \ldots v_{m}^{n}\right\}_{m \geq 1}$. If we call $\mathbb{F}$ to this full inclusion, we get another one $\mathbb{F}_{*}: \bmod \left(\mathbf{M}_{R}\left(\bar{T}_{n}\right)\right) \rightarrow \bmod \left(\mathbf{M}_{R}(\bar{T})\right)$ together with a retraction up to natural equivalence $\mathbb{F}^{*}: \bmod \left(\mathbf{M}_{R}(\bar{T})\right) \rightarrow \bmod \left(\mathbf{M}_{R}\left(\bar{T}_{n}\right)\right)$. Moreover, the first functor $\mathbb{F}_{*}$ restricts to the full subcategories of $\mathrm{f}$. p. modules, see Section 2, hence the decomposition problem for f. p. $R(\mathbf{n})$-modules is included in the decomposition problem for f. p. $R(E)$-modules, in particular we only need to prove Theorem 1.1 for card $E$ finite.

\section{Countably presented $R$-modules as finitely presented $\operatorname{RCFM}(R)$-MODULES}

There is a full exact inclusion of abelian categories

$$
\mathfrak{i}: \bmod (R) \longrightarrow \bmod (\operatorname{RCFM}(R))
$$

defined by $\mathfrak{i} M=\operatorname{Hom}_{R}\left(R\left\langle\mathbb{N}_{0}\right\rangle, M\right)$. The ring $\operatorname{RCFM}(R)$ acts on $\mathfrak{i} M$ by endomorphisms of $R\left\langle\mathbb{N}_{0}\right\rangle$. 
Let $\mathfrak{f}: \mathbf{M}_{R}\left(\bar{T}_{1}\right) \rightarrow \bmod (R)$ be the forgetful functor which sends a free $\bar{T}_{1}$ controlled $R$-module to its underlying $R$-module. The $\operatorname{RCFM}(R)$-module $\mathfrak{i} M$ can be regarded as the functor $\mathfrak{i} M=\operatorname{Hom}_{R}(\mathfrak{f}, M): \mathbf{M}_{R}\left(\bar{T}_{1}\right)^{o p} \rightarrow \mathbf{A b}$.

Proposition 4.1. The functor $\mathfrak{i}$ has an exact left-adjoint $\mathfrak{r}$ such that $\mathfrak{r} i$ is naturally equivalent to the identity functor. Moreover, $\mathfrak{r}$ can be chosen to be the evaluation functor in a free $\bar{T}_{1}$-controlled $R$-module with one generator.

Proof. Let $R\langle e\rangle_{\phi}$ be a free $\bar{T}_{1}$-controlled $R$-module whose basis is a singleton $\{e\}$ (all these objects are isomorphic in $\mathbf{M}_{R}\left(\bar{T}_{1}\right)$ by (3.2)). The endomorphism ring of this object is $R$, hence the evaluation functor $e v_{R\langle e\rangle_{\phi}}$ takes values in the category of $R$-modules. Let us see that the exact functor $e v_{R\langle e\rangle_{\phi}}$ is left-adjoint to i. A leftadjoint for $i$ exists and can be constructed by left additive Kan extension, see [12] 6, so we have just to check that $\operatorname{Hom}_{\mathbf{M}_{R}\left(\bar{T}_{1}\right)}\left(R\langle A\rangle_{\alpha}, \mathrm{i} M\right)=\operatorname{Hom}_{R}\left(e v_{R\langle e\rangle_{\phi}} R\langle A\rangle_{\alpha}, M\right)$ for any free $\bar{T}_{1}$-controlled $R$-module $R\langle A\rangle_{\alpha}$ in a natural way. This follows from the obvious natural identification $e v_{R\langle e\rangle_{\phi}} R\langle A\rangle_{\alpha}=R\langle A\rangle$ and Yoneda's lemma.

Corollary 4.2. If $M$ is an $R$-module and $\mathcal{N}$ an $\operatorname{RCFM}(R)$-module then there are natural isomorphisms $(n \geq 0)$

$$
\operatorname{Ext}_{R(E)}^{n}(\mathcal{N}, \mathfrak{i} M) \simeq \operatorname{Ext}_{R}^{n}(\mathfrak{r} \mathcal{N}, M) .
$$

In particular if $R=k$ is a field the $\operatorname{RCFM}(k)$-modules $\mathfrak{i} M$ are all injective.

An $R$-module is countably presented provided it is the cokernel of a morphism between free $R$-modules with countable basis. Obviously the cokernel of a morphism between countably presented $R$-modules is countably presented as well. In particular direct summands of countably presented $R$-modules are countably presented.

Proposition 4.3. The functor $\mathfrak{i}$ sends countably presented $R$-modules to finitely presented $\operatorname{RCFM}(R)$-modules.

In the proof of this proposition we shall use the row-column-finite matrices $\mathrm{A}$ and $\mathrm{B}$ defined by

- $\mathrm{a}_{i+1, i}=1\left(i \in \mathbb{N}_{0}\right)$ and $\mathrm{a}_{i j}=0$ in other cases,

- $\mathrm{b}_{\frac{n(n+1)}{2}+i, \frac{(n-1) n}{2}+i}=1$ for any $n>0$ and $0 \leq i<n$, and $\mathrm{b}_{i j}=0$ otherwise.

And we regard $\operatorname{RCFM}(R)$ as the endomorphism $R$-algebra of the free $\bar{T}_{1}$-controlled $R$-module $R\left\langle\mathbb{N}_{0}\right\rangle_{\delta}$ where $\delta: \mathbb{N}_{0} \subset[0,+\infty)$ is the inclusion, see Remark 3.9 and Proposition 3.4 .

Proof of (4.3). Since $\mathfrak{i}$ is exact it will be enough to check the proposition for the countably presented $R$-modules $R$ and $R\left\langle\mathbb{N}_{0}\right\rangle$. Recall that $\operatorname{Hom}_{\mathbf{M}_{R}\left(\bar{T}_{1}\right)}\left(R\left\langle\mathbb{N}_{0}\right\rangle_{\delta}, \mathfrak{i} M\right)=$ $\operatorname{Hom}_{R}\left(R\left\langle\mathbb{N}_{0}\right\rangle, M\right)$ for any $R$-module $M$. The $\operatorname{RCFM}(R)$-modules $\mathfrak{i} R$ and $\mathfrak{i} R\left\langle\mathbb{N}_{0}\right\rangle$ are the cokernels of $(\mathrm{I}-\mathrm{A})$ and $(\mathrm{I}-\mathrm{B})$ respectively. The natural projections onto the cokernel are given by the homomorphisms $R\left\langle\mathbb{N}_{0}\right\rangle \rightarrow R$ and $R\left\langle\mathbb{N}_{0}\right\rangle \rightarrow R\left\langle\mathbb{N}_{0}\right\rangle$ defined on generators by $n \mapsto 1(n \geq 0)$ and $\frac{n(n+1)}{2}+i \mapsto i(n \geq i \geq 0)$ respectively.

As a consequence of Propositions 4.1 and 4.3, and Remark 3.10 we get the next

Corollary 4.4. The representation problem for countably generated $R$-modules is contained into the representation problem for finitely presented $R(E)$-modules, where $E$ is any non-empty compact metrizable space. 


\section{A REVIEW ON PRO-CATEGORIES}

Any partially ordered set (poset) $\Lambda$ can be regarded as a small category with a unique morphism $u \rightarrow v$ provided $u \geq v, u, v \in \Lambda$. A poset $\Lambda$ is directed if given $u, v \in \Lambda$ there exists $w \in \Lambda$ with $w \geq u, v$. Moreover, $\Lambda$ is cofinite if the set $\{u \in \Lambda ; u \leq v\}$ is finite for every $v \in \Lambda$.

A pro-object or inverse system $X_{\bullet}$ over a category $\mathbf{C}$ is a functor $X_{\bullet}: \Lambda \rightarrow \mathbf{C}$ where $\Lambda$ is a directed cofinite poset. If $u \in \Lambda$ we usually write $X_{u}=X_{\bullet}(u)$. The morphisms $X_{\bullet}(u \rightarrow v)(u, v \in \Lambda, u \geq v)$ are the bonding morphisms of $X_{\bullet}$, and $\Lambda$ is the indexing set of the inverse system.

The category pro - $\mathbf{C}$ has objects inverse systems over $\mathbf{C}$. Morphism sets are given by the following formula

$$
\operatorname{Hom}_{\text {pro-C}}\left(X_{\bullet}, Y_{\bullet}\right)=\lim _{v} \operatorname{colim}_{u} \operatorname{Hom}_{\mathbf{C}}\left(X_{u}, Y_{v}\right) \text {. }
$$

We identify any object in $\mathbf{C}$ with the inverse system whose indexing set is a singleton $\Lambda=*$. This defines a full inclusion of categories $\mathbf{C} \subset$ pro $-\mathbf{C}$. This inclusion has a right-adjoint, the (inverse) limit functor $\lim$ : pro $-\mathbf{C} \rightarrow \mathbf{C}, \lim X \bullet$ $\lim _{u} X_{u}$.

The category pro $-\mathbf{C}$ is abelian whenever $\mathbf{C}$ is, see [6] 6.4. This will be always the case, because we are only going to use in this context the category $\mathbf{C}=\bmod (k)$ of $k$-vector spaces. If $V$ is a vector space and $X_{\bullet}$ an inverse system of vector spaces, then by (5.A)

$$
\operatorname{Hom}_{\operatorname{pro}-\bmod (k)}\left(V, X_{\bullet}\right)=\lim _{v} \operatorname{Hom}_{k}\left(V, X_{v}\right)=\operatorname{Hom}_{k}\left(V, \lim X_{\bullet}\right) .
$$

Hence, since $\operatorname{Hom}_{k}(V,-)$ is an exact functor in the category of vector spaces, the Grothendieck spectral sequence (see [10] 9.3) yields an isomorpshim

$$
\operatorname{Ext}_{\text {pro- } \bmod (k)}^{1}\left(V, X_{\bullet}\right)=\operatorname{Hom}_{k}\left(V, \lim ^{1} X_{\bullet}\right) .
$$

\section{Numerical inVARIANTS OF FINITELY PRESENTED $k(\boldsymbol{n})$-MOdULES}

In this section we shall define invariants of the isomorphism class of a f. p. $k(\mathbf{n})$ module lying in the abelian monoids $\mathbb{N}_{\infty, n}(n \geq 1)$. The abelian monoid $\mathbb{N}_{\infty, n}$ has $n+1$ generators

$$
1, \infty_{1}, \ldots \infty_{n}
$$

and $2 n$ relations

$$
1+\infty_{i}=\infty_{i}, \infty_{i}+\infty_{i}=\infty_{i}, \quad(1 \leq i \leq n)
$$

As a set $\mathbb{N}_{\infty, n}$ is

$$
\mathbb{N}_{\infty, n}=\mathbb{N}_{0} \sqcup\left\{\infty_{S}=\sum_{i \in S} \infty_{i} ; \emptyset \neq S \subset\{1, \ldots n\}\right\} .
$$

For the sake of simplicity if $n=1$ we write $\mathbb{N}_{\infty, 1}=\mathbb{N}_{\infty}$ and $\infty_{1}=\infty$.

Let $\varphi: k\langle B\rangle_{\beta} \rightarrow k\langle A\rangle_{\alpha}$ be a morphism in $\mathbf{M}_{k}\left(\bar{T}_{n}\right)$. We define the element $\lambda_{\varphi} \in \mathbb{N}_{\infty, n}$ in the following way: if the next vector space is finite-dimensional

$$
L_{\varphi}=\frac{k\langle A\rangle}{\bigcap_{m \geq 1} \sum_{i=1}^{n}\left[k\left\langle A_{m}^{i}\right\rangle+\varphi(k\langle B\rangle)\right]},
$$

then $\lambda_{\varphi}=\operatorname{dim} L_{\varphi}$, otherwise $\lambda_{\varphi}=\infty_{S}$, where $S \subset\{1, \ldots n\}$ is the biggest subset such that if $i \notin S$ then there exists $M \geq 1$ with $k\left\langle A_{M}^{i}\right\rangle \subset \varphi(k\langle B\rangle)$. 
Proposition 6.1. The element $\lambda_{\varphi}$ only depends on the isomorphism class of the f. p. $k(\boldsymbol{n})$-module Coker $\varphi$, and $\lambda_{\varphi \oplus \psi}=\lambda_{\varphi}+\lambda_{\psi}$.

Proof. One can readily check by using the alternative description of controlled homomorphisms given in Subsection 3.1 that the correspondence $\varphi \mapsto \Upsilon(\varphi)=L_{\varphi}$ defines an additive functor $\Upsilon$ from pair $\left(\mathbf{M}_{k}\left(\bar{T}_{n}\right)\right)$ to the category of $k$-vector spaces. Moreover, if $V_{\bullet}^{\varphi, i}$ is the inverse system of $k$-vector spaces indexed by $\mathbb{N}$ and given by $(1 \leq i \leq n)$

$$
V_{m}^{\varphi, i}=\frac{k\left\langle A_{m}^{i}\right\rangle+\varphi(k\langle B\rangle)}{\varphi(k\langle B\rangle)},
$$

and the obvious inclusions as bonding morphisms, the correspondences $\varphi \mapsto \Theta_{i}(\varphi)=$ $V_{\bullet}^{\varphi, i}$ also define additive functors $\Theta_{i}$ from $\operatorname{pair}\left(\mathbf{M}_{k}\left(\bar{T}_{n}\right)\right)$ to the pro-category of provector spaces. Furthermore, it is easy to see that the functors $\Upsilon, \Theta_{i}(1 \leq i \leq n)$ factor through the natural equivalence relation $\sim$ in $\operatorname{pair}\left(\mathbf{M}_{k}\left(\bar{T}_{n}\right)\right)$, hence the first statement of the proposition follows from (2.1) and the fact that $\lambda_{\varphi}$ is defined as $\operatorname{dim} \Upsilon(\varphi)$ provided this vector space is finite-dimensional, and otherwise $\lambda_{\varphi}=\infty_{S}$ where $S=\left\{i \in\{1, \ldots n\} ; \Theta_{i}(\varphi) \not 0\right\}$. The second part of the statement follows from the additivity of the functors $\Upsilon, \Theta_{i}(1 \leq i \leq n)$.

If the following vector space has finite dimension,

$$
M_{\varphi}^{i}=\frac{\bigcap_{m \geq 1}\left[k\left\langle A_{m}^{i}\right\rangle+\varphi(k\langle B\rangle)\right]}{\bigcap_{m \geq 1}\left\{\left[k\left\langle A_{m}^{i}\right\rangle+\varphi(k\langle B\rangle)\right] \cap\left[\sum_{j \neq i} k\left\langle A_{m}^{j}\right\rangle+\varphi(k\langle B\rangle)\right]\right\}},
$$

the element $\mu_{\varphi}^{i} \in \mathbb{N}_{\infty}(1 \leq i \leq n)$ is defined as $\mu_{\varphi}^{i}=\operatorname{dim} M_{\varphi}^{i}$, otherwise $\mu_{\varphi}^{i}=\infty$.

Proposition 6.2. The elements $\mu_{\varphi}^{i}(1 \leq i \leq n)$ only depend on the isomorphism class of the f. p. $k(\mathbf{n})$-module Coker $\varphi$, and $\mu_{\varphi \oplus \psi}^{i}=\mu_{\varphi}^{i}+\mu_{\psi}^{i}$.

Proof. By using the characterization of controlled homomorphisms given in Subsection 3.1 one readily checks that the correspondences $\varphi \mapsto M_{\varphi}^{i}$ define additive functors from pair $\left(\mathbf{M}_{k}\left(\bar{T}_{n}\right)\right)$ to the category of $k$-vector spaces. Moreover, these functors factor through the natural equivalence relation $\sim$, hence the proposition follows from (2.1).

In order to define elements $\nu_{\varphi}^{i} \in \mathbb{N}_{\infty}(1 \leq i \leq n)$ we introduce inverse systems of $k$-vector spaces $U_{\bullet}^{\varphi, i}$, indexed by the set $\mathbb{N} \times \mathbb{N}$ with the product partial order, given by

$$
U_{p q}^{\varphi, i}=\frac{k\left\langle A_{p}^{i}\right\rangle \cap \varphi(k\langle B\rangle)}{k\left\langle A_{p}^{i}\right\rangle \cap \varphi\left(k\left\langle B_{q}^{i}\right\rangle\right)},
$$

and bonding homomorphisms induced by the obvious inclusions of vector spaces. If the limit of $U_{\bullet}^{\varphi, i}$ is finite-dimensional we set $\nu_{\varphi}^{i}=\operatorname{dim} \lim U_{\bullet}^{\varphi, i}$, otherwise $\nu_{\varphi}^{i}=\infty$.

Proposition 6.3. The elements $\nu_{\varphi}^{i}(1 \leq i \leq n)$ only depend on the isomorphism class of the f. p. $k(\mathbf{n})$-module Coker $\varphi$, and $\nu_{\varphi \oplus \psi}^{i}=\nu_{\varphi}^{i}+\nu_{\psi}^{i}$.

Proof. One can check by using the description of controlled homomorphisms given in Subsection 3.1 that the correspondences $\varphi \mapsto U_{\bullet}^{\varphi, i}$ are additive functors from $\operatorname{pair}\left(\mathbf{M}_{k}\left(\bar{T}_{n}\right)\right)$ to the pro-category of pro-vector spaces, and these functors factor through the natural equivalence relation $\sim$, hence the proposition follows from (2.1). 
The former propositions of this section are summarized in the following

Corollary 6.4. There are well defined morphisms of abelian monoids $(n \in \mathbb{N})$

$$
\Phi_{n}: \operatorname{Iso}(\mathbf{f p}(k(\boldsymbol{n}))) \longrightarrow \mathbb{N}_{\infty, n} \times \prod_{i=1}^{n} \mathbb{N}_{\infty} \times \prod_{i=1}^{n} \mathbb{N}_{\infty}
$$

which send the isomorphism class $[\mathcal{M}]$ of a f. p. $k(\mathbf{n})$-module $\mathcal{M}=$ Coker $\varphi$ to

$$
\Phi_{n}([\mathcal{M}])=\left(\lambda_{\varphi},\left(\mu_{\varphi}^{i}\right)_{i=1}^{n},\left(\nu_{\varphi}^{i}\right)_{i=1}^{n}\right)
$$

From now on we shall write $\lambda_{\mathcal{M}}=\lambda_{\varphi}, \mu_{\mathcal{M}}^{i}=\mu_{\varphi}^{i}$ and $\nu_{\mathcal{M}}^{i}=\nu_{\varphi}^{i}(1 \leq i \leq n)$ if $\mathcal{M}=\operatorname{Coker} \varphi$ and omit the superindex $i$ when $n=1$.

Remark 6.5. There are $n$ full inclusions $\mathbb{F}^{i}: \mathbf{M}_{k}\left(\bar{T}_{1}\right) \rightarrow \mathbf{M}_{k}\left(\bar{T}_{n}\right)(1 \leq i \leq n)$ defined by identifying $T_{1}^{0}=\mathbb{N}_{0}$ (see (3.9)) with the subset $\left\{v_{0}\right\} \cup\left\{v_{m}^{i}\right\}_{m \geq 1} \subset T_{n}^{0}$ in the obvious way, see (3.10).

The next proposition can be easily checked by using the commutativity of (2.A) and the right-exactness of the functors $\mathbb{F}_{*}^{i}$.

Proposition 6.6. If $\mathcal{M}$ is a f. $p$. $k(\mathbf{1})$-module then for every $1 \leq i \leq n$

- $\lambda_{\mathbb{F}_{*}^{i} \mathcal{M}}=\lambda_{\mathcal{M}}$ if $\lambda_{\mathcal{M}} \in \mathbb{N}_{0}$, and $\lambda_{\mathbb{F}_{*}^{i} \mathcal{M}}=\infty_{i}$ otherwise,

- $\mu_{\mathbb{F}_{*}^{i} \mathcal{M}}^{i}=\mu_{\mathcal{M}}$ and $\mu_{\mathbb{F}_{*}^{i} \mathcal{M}}^{j}=0$ if $j \neq i$,

- $\nu_{\mathbb{F}_{*}^{i} \mathcal{M}}^{i}=\nu_{\mathcal{M}}$ and $\nu_{\mathbb{F}_{*}^{i} \mathcal{M}}^{j}=0$ if $j \neq i$.

\section{Classification of finitely PResented $\operatorname{RCFM}(k)$-Modules}

Recall from (3.9) that the $k$-algebra $k(\mathbf{1})$ coincides with $\operatorname{RCFM}(k)$, the $k$-algebra of matrices $\mathrm{A}=\left(\mathrm{a}_{i j}\right)_{i, j \in \mathbb{N}_{0}}$ with entries in $k$ such that every row and every column has at most a finite number of non-trivial entries (row-column-finite matrices). Those matrices are the endomorphisms of the free $\bar{T}_{1}$-controlled $k$-vector space $k\left\langle\mathbb{N}_{0}\right\rangle_{\delta}$, where $\delta: \mathbb{N}_{0} \subset[0,+\infty)$ is the inclusion of the vertex set. The unit element of the $k$-algebra $\operatorname{RCFM}(k)$ is the identity matrix I with $\mathrm{i}_{i i}=1\left(i \in \mathbb{N}_{0}\right)$ and $\mathrm{i}_{i j}=0$ if $i \neq j$. For the sake of simplicity we abbreviate $\mathcal{R}=\operatorname{RCFM}(k)$.

Consider the matrices A and B used in the proof of (4.3), they are defined as

- $\mathrm{a}_{i+1, i}=1\left(i \in \mathbb{N}_{0}\right)$ and $\mathrm{a}_{i j}=0$ in other cases,

- $\mathrm{b}_{\frac{n(n+1)}{2}+i, \frac{(n-1) n}{2}+i}=1$ for any $n>0$ and $0 \leq i<n$, and $\mathrm{b}_{i j}=0$ otherwise.

We define the following $\mathcal{R}$-modules

- $\mathcal{A}=\frac{\mathcal{R}}{\mathrm{A} \mathcal{R}}$,

- $\mathcal{B}=\frac{\mathcal{R}}{(1-\mathrm{A}) \mathcal{R}}$,

- $\mathrm{C}=\frac{\mathcal{R}}{\left(1-\mathrm{A}^{t}\right) \mathcal{R}}$

- $\mathcal{B}_{\infty}=\frac{\mathcal{R}}{(\mathrm{I}-\mathrm{B}) \mathcal{R}}$,

- $\mathcal{C}_{\infty}=\frac{\mathcal{R}}{\left(1-\mathrm{B}^{t}\right) \mathcal{R}}$.

The main result of this section is the next

Theorem 7.1 (Classification of f. p. $\operatorname{RCFM}(k)$-modules). There is a solution to the decomposition problem in the category of $f . p . \operatorname{RCFM}(k)$-modules given by the following elementary modules

$$
\mathcal{A}, \mathcal{R}, \mathcal{B}, \mathcal{B}_{\infty}, \mathcal{C}, \mathcal{C}_{\infty},
$$


and elementary isomorphisms

$$
\begin{gathered}
\mathcal{A} \oplus \mathcal{R} \simeq \mathcal{R}, \quad \mathcal{R} \oplus \mathcal{R} \simeq \mathcal{R}, \mathcal{B} \oplus \mathcal{B}_{\infty} \simeq \mathcal{B}_{\infty} \\
\mathcal{B}_{\infty} \oplus \mathcal{B}_{\infty} \simeq \mathcal{B}_{\infty}, \quad \mathcal{C} \oplus \mathcal{C}_{\infty} \simeq \mathcal{C}_{\infty}, \quad \mathcal{C}_{\infty} \oplus \mathcal{C}_{\infty} \simeq \mathcal{C}_{\infty} .
\end{gathered}
$$

This theorem implies Theorems 1.1 and 1.2 for card $E=1$. It is a direct consequence of the next two results. We shall use the following notation, given $n \in \mathbb{N}_{0}$ we write $\mathcal{A}_{n}, \mathcal{B}_{n}$ and $\mathcal{C}_{n}$ for the direct sum of $n$ copies of $\mathcal{A}, \mathcal{B}$ or $\mathcal{C}$, respectively, and $\mathcal{A}_{\infty}=\mathcal{R}$.

Theorem 7.2. For every f. p. R-module $\mathcal{M}$ there is an isomorphism

$$
\mathcal{M} \simeq \mathcal{A}_{\lambda_{\mathcal{M}}} \oplus \mathcal{B}_{\mu_{\mathcal{M}}} \oplus \mathcal{C}_{\nu_{\mathcal{M}}}
$$

Proposition 7.3. We have the following equalities:

- $\Phi_{1}(\mathcal{A})=(1,0,0)$,

- $\Phi_{1}(\mathcal{B})=(0,1,0)$,

- $\Phi_{1}(\mathcal{C})=(0,0,1)$,

- $\Phi_{1}(\mathcal{R})=(\infty, 0,0)$,

- $\Phi_{1}\left(\mathcal{B}_{\infty}\right)=(0, \infty, 0)$,

- $\Phi_{1}\left(\mathcal{C}_{\infty}\right)=(0,0, \infty)$.

In particular we have that

Corollary 7.4. The monoid morphism

$$
\Phi_{1}: \operatorname{Iso}(\mathbf{f p}(k(\mathbf{1}))) \longrightarrow \mathbb{N}_{\infty} \times \mathbb{N}_{\infty} \times \mathbb{N}_{\infty}
$$

is an isomorphism.

The proof of Proposition 7.3 will be given later. Theorem 7.2 is a direct consequence of Lemmas 7.18, 7.19, 7.20 and 7.22. These are some of the hardest technical results of this paper. In fact the rest of this long section is highly technical. It is focused towards proving Theorem 7.2, although some interesting corollaries on the homological algebra of finitely presented $\mathcal{R}$-modules are derived from the technical lemmas. These homological results are used in the proof of (7.2) as well as in the appendix. We advice the reader to skip this material in a first reading.

The next result is an easy computation.

Lemma 7.5. The following equalities hold in $\mathcal{R}$ :

(1) $A^{t} A=I$,

(2) $\mathrm{B}^{t} \mathrm{~B}=\mathrm{I}$.

The next lemma follows directly from (3.2).

Lemma 7.6. Two free $\bar{T}_{1}$-controlled $k$-vector spaces $k\langle A\rangle_{\alpha}, k\langle B\rangle_{\beta}$ are isomorphic in $\mathbf{M}_{k}\left(\bar{T}_{1}\right)$ if and only if $A$ and $B$ have the same cardinal.

Lemma 7.7. The $\mathcal{R}$-module $\mathcal{A}$ is isomorphic to any 1 -dimensional free $\bar{T}_{1}$-controlled $k$-vector space.

Proof. If $k\langle e\rangle_{\phi}$ is a 1 -dimensional free $\bar{T}_{1}$-controlled $k$-vector space, the cokernel of $\mathrm{A}$ is given by the controlled homomorphism $\varphi: k\left\langle\mathbb{N}_{0}\right\rangle_{\delta} \rightarrow k\langle e\rangle_{\phi}$ defined over the basic elements as $0 \mapsto e$ and $n \mapsto 0$ for $n>0$.

The proof of Proposition 7.3 is as follows. 
Proof of 7.3. In this proof we omit some straightforward but tedious computations which can be carried out by the interested reader with not too much difficulty. We shall write $\mathbb{N}_{p}(p \geq 1)$ for the set of naturals $\geq p$.

The $\mathcal{R}$-module $\mathcal{R}$ corresponds to the free $\overline{\bar{T}}_{1}$-controlled $k$-vector space $k\left\langle\mathbb{N}_{0}\right\rangle_{\delta}$ where $\delta: \mathbb{N}_{0} \subset[0,+\infty)$ is the inclusion, hence it is the cokernel of the trivial morphism 0: $0 \rightarrow k\left\langle\mathbb{N}_{0}\right\rangle_{\delta}$, and the equalities $\lambda_{\mathcal{R}}=\infty, \mu_{\mathcal{R}}=0$ hold immediately, moreover, $U_{m, n}^{0}=k\left\langle\mathbb{N}_{m}\right\rangle$ for all $m, n \in \mathbb{N}$, and given $M \geq m, N \geq n$ the corresponding bonding homomorphism in $U_{\bullet}^{0}$ is the inclusion $U_{M, N}^{0}=k\left\langle\mathbb{N}_{M}\right\rangle \subset k\left\langle\mathbb{N}_{m}\right\rangle=U_{m, n}^{0}$, therefore $\lim U_{\bullet}^{0}=\bigcap_{m \in \mathbb{N}} k\left\langle\mathbb{N}_{m}\right\rangle=0$ and $\nu_{\mathcal{R}}=0$.

By (7.7) the $\mathbf{M}_{k}\left(\bar{T}_{1}\right)$-module $\mathcal{A}$ is the cokernel of the trivial morphism $0 \rightarrow k\langle e\rangle_{\phi}$ where $k\langle e\rangle_{\phi}$ is a 1-dimensional free $\bar{T}_{1}$-controlled $k$-vector space, hence the equality $\Phi_{1}(\mathcal{A})=(1,0,0)$ follows easily.

One can check that $k\left\langle\mathbb{N}_{n}\right\rangle+(\mathbf{I}-\mathrm{A})\left(k\left\langle\mathbb{N}_{0}\right\rangle\right)=k\left\langle\mathbb{N}_{0}\right\rangle$ for all $n \in \mathbb{N}$, and $k\left\langle\mathbb{N}_{0}\right\rangle /(\mathbf{I}-$ A) $\left(k\left\langle\mathbb{N}_{0}\right\rangle\right) \simeq k$ generated by the class of any $n \in \mathbb{N}_{0}$, so $\lambda_{\mathcal{B}}=0$ and $\mu_{\mathcal{B}}=1$. Moreover, $k\left\langle\mathbb{N}_{n}\right\rangle \cap(\mathbf{I}-\mathbf{A})\left(k\left\langle\mathbb{N}_{0}\right\rangle\right)=(\mathbf{I}-\mathrm{A})\left(k\left\langle\mathbb{N}_{n}\right\rangle\right)$ and hence $U_{n, n}^{(I-A)}=0$ for all $n \in \mathbb{N}$, therefore $\lim U_{\bullet}^{(\mathrm{I}-\mathrm{A})}=0$ since the diagonal subset $\{(n, n) ; n \in \mathbb{N}\} \subset \mathbb{N} \times \mathbb{N}$ is cofinal, so $\nu_{\mathcal{B}}=0$.

One can see that $\left(\mathrm{I}-\mathrm{A}^{t}\right)\left(k\left\langle\mathbb{N}_{0}\right\rangle\right)=k\left\langle\mathbb{N}_{0}\right\rangle$, hence $\lambda_{\mathcal{C}}=0=\mu_{\mathcal{E}}$, moreover $\left(\mathbf{I}-\mathrm{A}^{t}\right)\left(k\left\langle\mathbb{N}_{n}\right\rangle\right)$ is generated by the set $\{m-(m-1)\}_{m \geq n}$, therefore $U_{n-1, n}^{\left(\mathbf{I}-\mathrm{A}^{t}\right)} \simeq k$ generated by the class of any $m \geq n-1$ and the bonding homomorphism $U_{n, n+1}^{\left(\mathrm{I}-\mathrm{A}^{t}\right)} \rightarrow$ $U_{n-1, n}^{\left(\mathrm{I}-\mathrm{A}^{t}\right)}$ is an isomorphism, so again by cofinality we see that $\lim U_{\bullet}^{\left(\mathrm{I}-\mathrm{A}^{t}\right)} \simeq k$, in particular $\nu_{\mathrm{e}}=1$.

The vector space $k\left\langle\mathbb{N}_{n}\right\rangle+(\mathrm{I}-\mathrm{B})\left(k\left\langle\mathbb{N}_{0}\right\rangle\right)$ is the whole $k\left\langle\mathbb{N}_{0}\right\rangle$, so $\lambda_{\mathcal{B}_{\infty}}=0$, moreover, a basis of $k\left\langle\mathbb{N}_{0}\right\rangle /(\mathrm{I}-\mathrm{B})\left(k\left\langle\mathbb{N}_{0}\right\rangle\right)$ is $\left\{\frac{n(n+3)}{2}\right\}_{n \in \mathbb{N}_{0}}$, hence $\mu_{\mathcal{B}_{\infty}}=\infty$. One can check that $k\left\langle\mathbb{N}_{n}\right\rangle \cap(\mathrm{I}-\mathrm{B})\left(k\left\langle\mathbb{N}_{0}\right\rangle\right)=(\mathbf{I}-\mathrm{B})\left(k\left\langle\mathbb{N}_{n}\right\rangle\right)$, therefore $U_{n, n}^{(\mathrm{I}-\mathrm{B})}=0$, $\lim U_{\bullet}^{(1-\mathrm{B})}=0$ and $\nu_{\mathcal{B}_{\infty}}=0$.

Finally $\left(\mathrm{I}-\mathrm{B}^{t}\right)\left(k\left\langle\mathbb{N}_{0}\right\rangle\right)=k\left\langle\mathbb{N}_{0}\right\rangle$, so $\lambda_{\mathcal{C}_{\infty}}=0=\mu_{\mathcal{C}_{\infty}}$, and there are isomorphisms $\left(n \in \mathbb{N}_{0}\right)$

$$
U_{\frac{(n+1)(n+2)}{2}, \frac{n(n+1)}{2}}^{\left(\mathrm{I}-\mathrm{B}^{t}\right)} \simeq k\left\langle\frac{n(n+1)}{2}, \ldots \frac{n(n+1)}{2}+n\right\rangle,
$$

moreover, the following bonding homomorphism $(n>0)$

$$
U_{\frac{(n+1)(n+2)}{2}, \frac{n(n+1)}{2}}^{\left(1-\mathrm{B}^{t}\right)} \longrightarrow U_{\frac{n(n+1)}{2}}^{\left(1-\mathrm{B}^{t}\right)}, \frac{(n-1) n}{2}
$$

sends $\frac{n(n+1)}{2}+m$ to $\frac{(n-1) n}{2}+m$ if $m<n$ and $\frac{n(n+1)}{2}+n$ to the trivial element, so $\lim U_{\bullet}^{\left(1-\mathrm{B}^{t}\right)}=\prod_{\mathbb{N}_{0}} k$ is the direct product of an infinite countable number of copies of $k$ and the equality $\nu_{\mathcal{C}_{\infty}}=\infty$ holds.

Lemma 7.8. There is an $\mathcal{R}$-module isomorphism $\frac{\mathcal{R}}{\mathrm{BR}} \simeq \mathcal{R}$.

Proof. Let $A \subset \mathbb{N}_{0}$ be the infinite subset $A=\left\{\frac{n(n+3)}{2}\right\}_{n \in \mathbb{N}_{0}}$, and $\alpha: A \subset \mathbb{N}_{0}$ the inclusion. The next sequence, where $\varphi$ is the obvious projection, is exact

$$
k\left\langle\mathbb{N}_{0}\right\rangle_{\delta} \stackrel{\mathrm{B}}{\hookrightarrow} k\left\langle\mathbb{N}_{0}\right\rangle_{\delta} \stackrel{\varphi}{\rightarrow} k\langle A\rangle_{\alpha} .
$$

Hence the lemma follows from (7.6). 
Lemma 7.9. Left-multiplication by one of the following matrices induces an injective right- $\mathcal{R}$-module homomorphism $\mathcal{R} \rightarrow \mathcal{R}$,

$$
A,(I-A),\left(I-A^{t}\right),(I-B),\left(I-B^{t}\right) \text {. }
$$

Proof. The matrix A has a left-inverse in $\mathcal{R}$ by (7.5). The other matrices have a left-inverse either in the $k$-algebra $\operatorname{CFM}(k)$ of column-finite matrices or in the $k$-algebra $\operatorname{RFM}(k)$ of row-finite matrices. Both $k$-algebras contain $\mathcal{R}$, moreover, $\mathcal{R}=\operatorname{CFM}(k) \cap \operatorname{RFM}(k)$. The $k$-algebra $\operatorname{CFM}(k)$ is just the endomorphism ring of the $k$-vector space $k\left\langle\mathbb{N}_{0}\right\rangle$, and there is an isomorphism $\operatorname{RFM}(k) \simeq \operatorname{CFM}(k)^{o p}$ given by transposition. More precisely, let $\mathrm{C}, \mathrm{D}$ be the matrices in $\operatorname{RFM}(k)$ defined by $\mathrm{c}_{i j}^{1}=1$ if $i \geq j$ and zero otherwise, and $\mathrm{d}_{\frac{(m-1) m}{2}+i, \frac{(n-1) n}{2}+i}=1(m \geq n>i \geq 0)$ and trivial in other cases. One can check that $\mathrm{C}^{2}(\mathrm{I}-\mathrm{A}) \stackrel{2}{=} \mathrm{I}, \mathrm{C}^{t}\left(\mathrm{I}-\mathrm{A}^{t}\right)=\mathrm{I}, \mathrm{D}(\mathrm{I}-\mathrm{B})=\mathrm{I}$ and $\mathrm{D}^{t}\left(\mathrm{I}-\mathrm{B}^{t}\right)=\mathrm{I}$, hence the lemma follows.

Proposition 7.10. There are extensions of $\mathcal{R}$-modules

(1) $\mathcal{A} \hookrightarrow \mathcal{B} \rightarrow \mathcal{C}$,

(2) $\mathcal{R} \hookrightarrow \mathcal{B}_{\infty} \rightarrow \mathcal{C}_{\infty}$.

Proof. By using (7.5), (7.8) and (7.9) we get the following equalities, isomorphisms and short exact sequences, which correspond to the extensions of the statement

$$
\begin{gathered}
\frac{\mathcal{R}}{\mathrm{A} \mathcal{R}} \simeq \frac{\left(\mathrm{I}-\mathrm{A}^{t}\right) \mathcal{R}}{\left(\mathrm{I}-\mathrm{A}^{t}\right) \mathrm{A} \mathcal{R}}=\frac{\left(\mathrm{I}-\mathrm{A}^{t}\right) \mathcal{R}}{(\mathrm{I}-\mathrm{A}) \mathcal{R}} \hookrightarrow \frac{\mathcal{R}}{(\mathrm{I}-\mathrm{A}) \mathcal{R}} \rightarrow \frac{\mathcal{R}}{\left(I-\mathrm{A}^{\mathrm{t}}\right) \mathcal{R}}, \\
\mathcal{R} \simeq \frac{\mathcal{R}}{\mathrm{B} \mathcal{R}} \simeq \frac{\left(\mathrm{I}-\mathrm{B}^{t}\right) \mathcal{R}}{\left(\mathrm{I}-\mathrm{B}^{t}\right) \mathrm{B} \mathcal{R}}=\frac{\left(\mathrm{I}-\mathrm{B}^{t}\right) \mathcal{R}}{(\mathrm{I}-\mathrm{B}) \mathcal{R}} \hookrightarrow \frac{\mathcal{R}}{(\mathrm{I}-\mathrm{B}) \mathcal{R}} \rightarrow \frac{\mathcal{R}}{\left(I-\mathrm{B}^{\mathrm{t}}\right) \mathcal{R}} .
\end{gathered}
$$

The proof of the following proposition is contained in the proof of (4.3).

Proposition 7.11. Given a k-vector space $V$, if $\operatorname{dim} V<\aleph_{0}$ then $\mathfrak{i} V=\mathcal{B}_{\operatorname{dim} V}$, and $\mathfrak{i} V=\mathcal{B}_{\infty}$ if $\operatorname{dim} V=\aleph_{0}$.

The next corollary follows from (7.11) and (4.2).

Corollary 7.12. The $\mathcal{R}$-module $\mathcal{B}_{d}$ is injective for every $d \in \mathbb{N}_{\infty}$.

In the next lemma we show that one can adapt the basis of a countably generated vector space to a decreasing filtration.

Lemma 7.13. Let $V_{0} \supset V_{1} \supset \cdots \supset V_{n} \supset V_{n+1} \supset \cdots$ be a decreasing sequence of $k$-vector spaces such that $V_{0}$ is the union of an increasing sequence of finitedimensional subspaces $V_{0}^{0} \subset V_{0}^{1} \subset \cdots \subset V_{0}^{n} \subset V_{0}^{n+1} \subset \cdots, V_{0}=\bigcup_{n \in \mathbb{N}_{0}} V_{0}^{n}$. If we set $V_{0}^{-1}=0, V_{m}^{n}=V_{0}^{n} \cap V_{m}\left(n+1, m \in \mathbb{N}_{0}\right), V_{\infty}=\bigcap_{n \in \mathbb{N}_{0}} V_{n}$ and choose (finite and possibly empty) sets $\left\{a_{n m}^{l} ; 1 \leq l \leq r_{n m}\right\} \subset V_{m}^{n}$ such that the sets

$$
\left\{a_{n m}^{l}+\left(V_{m}^{n-1}+V_{m+1}^{n}\right) ; 1 \leq l \leq r_{n m}\right\}
$$

are basis of $V_{m}^{n} /\left(V_{m}^{n-1}+V_{m+1}^{n}\right)\left(n, m \in \mathbb{N}_{0}\right)$, then given $m, n, p \in \mathbb{N}_{0}$ with $m \leq p$

(1) $\left\{a_{i j}^{l_{i j}}+V_{p}^{n} ; i \leq n, m \leq j<p, 1 \leq l_{i j} \leq r_{i j}\right\}$ is a basis of $V_{m}^{n} / V_{p}^{n}$,

(2) $\left\{a_{i j}^{l_{i j}}+V_{\infty} ; i \leq n, m \leq j, 1 \leq l_{i j} \leq r_{i j}\right\}$ is a basis of $\left(V_{m}^{n}+V_{\infty}\right) / V_{\infty}$,

(3) $\left\{a_{i j}^{l_{i j}}+V_{\infty} ; i \in \mathbb{N}_{0}, m \leq j, 1 \leq l_{i j} \leq r_{i j}\right\}$ is a basis of $V_{m} / V_{\infty}$. 
Proof. Since $V_{0}^{n}$ is a finite-dimensional vector space it is artinian and the decreasing sequence $V_{0}^{n} \supset V_{1}^{n} \supset \cdots \supset V_{m}^{n} \supset V_{m+1}^{n} \supset \cdots$ stabilizes, it is, there exists $M_{n} \in \mathbb{N}_{0}$ such that $V_{m}^{n}=V_{M_{n}}^{n}$ for every $m \geq M_{n}$, in particular $V_{M_{n}}^{n}=V_{0}^{n} \cap V_{\infty}$. If we choose for every $n \in \mathbb{N}_{0}$ the minimum $M_{n}$ satisfying this condition then $M_{n} \leq M_{n+1}$ since

$$
V_{M_{n+1}}^{n}=V_{M_{n+1}}^{n} \cap V_{M_{n+1}}^{n+1}=V_{0}^{n} \cap V_{M_{n+1}} \cap V_{0}^{n+1} \cap V_{\infty}=V_{0}^{n} \cap V_{\infty}=V_{M_{n}}^{n} .
$$

Notice that (1) is trivial for $m \geq M_{n}$ since $V_{m}^{n}=V_{p}^{n}=V_{M_{n}}^{n}$ and $\left\{a_{i j}^{l} ; 1 \leq l \leq r_{i j}\right\}=$ $\emptyset$ whenever $p \geq m \geq M_{n}, i \leq n$ and $j \geq M_{n}$. Therefore the unique elements $(n, m, p)$ for which we still have to check (1) lie in the set $S=\left\{(n, m, p) ; n \in \mathbb{N}_{0}, 0 \leq m \leq M_{n}, p \geq m\right\}$. Let us order this set in the following way

$$
(n, m, p) \leq\left(n^{\prime}, m^{\prime}, p^{\prime}\right) \Leftrightarrow\left\{\begin{array}{l}
n<n^{\prime} \\
\text { or } \\
n=n^{\prime} \text { and } m>m^{\prime} \\
\text { or } \\
n=n^{\prime}, m=m^{\prime} \text { and } p \leq p^{\prime} .
\end{array}\right.
$$

One readily checks that this is a well order on $S$, since the second coordinate has an upper bound (depending on the first one). The minimum of $S$ is $\left(0, M_{0}, M_{0}\right)$, moreover if $m<M_{n}$ the element $(n, m, m)$ is the least upper bound of $\{(n, m+1, p) ; p>m\}$, and given $n>0$ the element $\left(n, M_{n}, M_{n}\right)$ is the least upper bound of the set $\left\{(n-1, m, p) ; m \leq M_{n-1}, p \geq m\right\}$. Any other element in $S$ is a successor. We have already checked (1) for the elements $\left(n, M_{n}, p\right) \in S$, moreover, it is trivial for $(n, m, m) \in S$, hence (1) holds for the minimum and all limit elements in $S$. A generic successor in $S$ has the form $(n, m, p+1)$ for some $p \geq m$. Notice that we have already check (1) for some successors as well, namely for those with $m=M_{n}$. We are now going to proceed by induction, it is, we shall prove (1) for every successor in $S$ with $m<M_{n}$ supposing that (1) holds for all the strictly lower elements. We are going to distinguish three cases:

For $(0, m, p+1)(1)$ follows from the exactness of the sequence

$$
\frac{V_{p}^{0}}{V_{p+1}^{0}} \hookrightarrow \frac{V_{m}^{0}}{V_{p+1}^{0}} \rightarrow \frac{V_{m}^{0}}{V_{p}^{0}}
$$

the equality $V_{0}^{-1}=0$ and the inequality $(0, m, p)<(0, m, p+1)$, and the inequality $(0, p+1, p)<(0, m, p+1)$ if $p<M_{0}$ or the equalities $V_{p}^{0}=V_{p+1}^{0}=V_{M_{0}}^{0}$ and $\left\{a_{0 p}^{l} ; 1 \leq l \leq r_{0 p}\right\}=\emptyset$ if $p \geq M_{0}$.

For $(n, m, m+1)$ with $n>0$ (1) is a consequence of the exactness of the sequence

$$
\frac{V_{m}^{n-1}+V_{m+1}^{n}}{V_{m+1}^{n}} \hookrightarrow \frac{V_{m}^{n}}{V_{m+1}^{n}} \rightarrow \frac{V_{m}^{n}}{V_{m}^{n-1}+V_{m+1}^{n}},
$$

the obvious isomorphism

$$
\frac{V_{m}^{n-1}}{V_{m+1}^{n-1}} \simeq \frac{V_{m}^{n-1}+V_{m+1}^{n}}{V_{m+1}^{n}}
$$

the inequality $(n-1, m, m+1)<(n, m, m+1)$ if $m<M_{n-1}$, or the equalities $V_{m}^{n-1}=V_{m+1}^{n-1}=V_{M_{n-1}}^{n-1}$ and $\left\{a_{i n} ; 1 \leq l \leq r_{i n}\right\}=\emptyset$ if $i \leq n-1$ and $m \geq M_{n-1}$. 
For $(n, m, p+1)$ with $n>0$ and $p>m$ (1) follows from the exactness of the sequence

$$
\frac{V_{p}^{n}}{V_{p+1}^{n}} \hookrightarrow \frac{V_{m}^{n}}{V_{p+1}^{n}} \rightarrow \frac{V_{m}^{n}}{V_{p}^{n}}
$$

the inequality $(n, m, p)<(n, m, p+1)$, and the inequality $(n, p, p+1)<(n, m, p+1)$ if $p<M_{n}$ or the equalities $V_{p}^{n}=V_{p+1}^{n}=V_{M_{n}}^{n}$ and $\left\{a_{i p}^{l} ; 1 \leq l \leq r_{i p}\right\}=\emptyset$ if $i \leq n$ and $p \geq M_{n}$.

Once we have seen that (1) holds, (2) is a consequence of (1) for $p=M_{n}$, the isomorphism $\left(V_{m}^{n}+V_{\infty}\right) / V_{\infty} \simeq V_{m}^{n} / V_{M_{n}}^{n}$, and the fact that $\left\{a_{i j}^{l} ; 1 \leq l \leq r_{i j}\right\}=\emptyset$ is the empty set for $i \leq n$ and $j \geq M_{n}$. Finally (3) follows from (2) and the equality $V_{m}=\bigcup_{n \in \mathbb{N}_{0}} V_{m}^{n}$.

The next proposition is an interesting consequence of the previous lemma. It does not hold in general when the ground ring is not a field, compare [1].

Proposition 7.14. The image of a morphism between finitely generated free $\mathbf{M}_{k}\left(\bar{T}_{1}\right)$ modules is finitely generated free.

Proof. Let $\varphi: k\langle B\rangle_{\beta} \rightarrow k\langle A\rangle_{\alpha}$ be a morphism in $\mathbf{M}_{k}\left(\bar{T}_{1}\right)$. If we define the $k$-vector spaces $V_{0}=\varphi(k\langle B\rangle), V_{n}=\varphi\left(k\left\langle B_{n}\right\rangle\right) \subset k\langle A\rangle(n \in \mathbb{N})$ and $V_{0}^{n}=\varphi\left(k\left\langle_{n} B\right\rangle\right)(n \geq 0)$ we can apply Lemma 7.13. Moreover, with the notation of that lemma $V_{\infty}=0$ since for every $n \geq 1$ there exists $N_{n} \geq 1$ such that $V_{N_{n}} \subset k\left\langle A_{n}\right\rangle$ and $\cap_{n \geq 1} k\left\langle A_{n}\right\rangle=$ 0 . We define the set $\underline{B}=\left\{a_{i j}^{l} ; i, j \in \mathbb{N}_{0}, 1 \leq l \leq r_{i j}\right\}$ and the function $\underline{\beta}: \underline{B} \rightarrow$ $\mathbb{N}_{0} \subset[0,+\infty)$ by $\underline{\beta}\left(a_{n m}^{l}\right)=m$. By (7.13) (3) the set $\underline{B}$ is a basis of $V_{0}$ and $\underline{B}_{m}$ a basis of $V_{m} \overline{(m \geq 1)}$ since $V_{\infty}=0$. The function $\beta$ is a height function because the cardinal of $\beta^{-1}(m)$ is $\operatorname{dim} V_{m} / V_{m+1}<\aleph_{0}\left(m \in \mathbb{N}_{0}\right)$. Moreover, the inclusion $k\langle\underline{B}\rangle=\varphi(k\langle\bar{B}\rangle) \subset k\langle A\rangle$ and the projection $k\langle B\rangle \rightarrow \varphi(k\langle B\rangle)=k\langle\underline{B}\rangle$ give rise to controlled homomorphisms $k\langle\underline{B}\rangle_{\underline{\beta}} \hookrightarrow k\langle A\rangle_{\alpha}$ and $k\langle B\rangle_{\beta} \rightarrow k\langle\underline{B}\rangle_{\underline{\beta}}$ which are an $\mathbf{M}_{k}\left(\bar{T}_{1}\right)$-module monomorphism and epimorphism respectively and their composition is $\varphi$, hence $k\langle\underline{B}\rangle_{\underline{\beta}}$ together with these morphisms is the image of $\varphi$.

Corollary 7.15. Any finitely presented $\mathbf{M}_{k}\left(\bar{T}_{1}\right)$-module is the cokernel of a monomorphism between finitely generated free $\mathbf{M}_{k}\left(\bar{T}_{1}\right)$-modules.

Corollary 7.16. Finitely presented $\mathcal{R}$-modules have projective dimension $\leq 1$.

Corollary 7.17. We have $\operatorname{Ext}^{1}\left(\mathcal{M}, \mathcal{C}_{d}\right)=0$ for any f. $p$. $\mathcal{R}$-module $\mathcal{M}$ and $d \in \mathbb{N}_{\infty}$.

Proof. By (7.16) the functor $\operatorname{Ext}^{1}(\mathcal{M},-)$ is right-exact, hence the corollary follows from (7.10) and (7.12).

Now we begin with the lemmas which prove Theorem 7.2.

Lemma 7.18. Given any $f$. $p$. $\mathcal{R}$-module $\mathcal{M}$, there exists another $f . p$. $\mathcal{R}$-module $\mathcal{N}$ with $\lambda_{\mathcal{N}}=0$ such that $\mathcal{N} \simeq \mathcal{A}_{\lambda_{\mathcal{M}}} \oplus \mathcal{N}$.

Proof. Suppose that $\mathcal{N}=$ Coker $\varphi$ for some $\varphi: k\langle B\rangle_{\beta} \rightarrow k\langle A\rangle_{\alpha}$ in $\mathbf{M}_{k}\left(\bar{T}_{1}\right)$. Let us consider the decreasing sequence of $k$-vector spaces given by $V_{0}=k\langle A\rangle / \varphi(k\langle B\rangle)$ and

$$
V_{n}=\frac{k\left\langle A_{n}\right\rangle+\varphi(k\langle B\rangle)}{\varphi(k\langle B\rangle)}, n \in \mathbb{N} .
$$


The vector space $V_{0}$ is the union of the following sequence of finite-dimensional $k$-vector spaces $\left(n \in \mathbb{N}_{0}\right)$

$$
V_{0}^{n}=\frac{k\left\langle_{n} A\right\rangle+\varphi(k\langle B\rangle)}{\varphi(k\langle B\rangle)} .
$$

If $\left\{a_{n m}^{l} ; 1 \leq l \leq r_{n m}\right\} \subset V_{m}^{n}$ is a set as in (7.13) we can suppose that $a_{n m}^{l}=$ $e_{n m}^{l}+\varphi(k\langle B\rangle)$ for some $\left.e_{n m}^{l} \in k{ }_{n} A_{m}\right\rangle$, here we use the next obvious isomorphism

$$
\frac{k\left\langle_{n} A_{m}\right\rangle}{\left.k{ }_{n} A_{m}\right\rangle \cap \varphi(k\langle B\rangle)} \simeq \frac{k\left\langle_{n} A_{m}\right\rangle+\varphi(k\langle B\rangle)}{\varphi(k\langle B\rangle)}=V_{m}^{n} .
$$

We consider the set $C=\left\{a_{n m}^{l_{n m}}+V_{\infty} ; n, m \in \mathbb{N}_{0}, 1 \leq l_{n m} \leq r_{n m}\right\}$ and the function $\gamma: C \rightarrow \mathbb{N}_{0} \subset[0,+\infty)$ with $\gamma\left(a_{n m}^{l_{n m}}+V_{\infty}\right)=m$. This function is a height function, since the set $\gamma^{-1}(m)=\left\{a_{n m}^{l_{n m}}+V_{\infty} ; n \in \mathbb{N}_{0}, 1 \leq l_{n m} \leq r_{n m}\right\}$ is bijective with a basis of $V_{m} / V_{m+1}$ by (7.13) (3), and we have the following surjection and isomorphisms

$$
\frac{k\left\langle A_{m}\right\rangle}{k\left\langle A_{m+1}\right\rangle} \rightarrow \frac{k\left\langle A_{m}\right\rangle}{k\left\langle A_{m+1}\right\rangle+\left[\varphi(k\langle B\rangle) \cap k\left\langle A_{m}\right\rangle\right]} \simeq \frac{k\left\langle A_{m}\right\rangle+\varphi(k\langle B\rangle)}{k\left\langle A_{m+1}\right\rangle+\varphi(k\langle B\rangle)} \simeq \frac{V_{m}}{V_{m+1}},
$$

and $\operatorname{dim} k\left\langle A_{m}\right\rangle / k\left\langle A_{m+1}\right\rangle=\operatorname{card} \alpha^{-1}(m)<\aleph_{0}$. The underlying $k$-vector space of $k\langle C\rangle_{\gamma}$ is $V_{0} / V_{\infty}$, moreover, the natural projection

$$
k\langle A\rangle \rightarrow \frac{k\langle A\rangle}{\bigcap_{n \geq 1}\left[k\left\langle A_{n}\right\rangle+\varphi(k\langle B\rangle)\right]} \simeq \frac{V_{0}}{V_{\infty}}=k\langle C\rangle
$$

give rise to a $\bar{T}_{1}$-controlled homomorphism $v_{0}: k\langle A\rangle_{\alpha} \rightarrow k\langle C\rangle_{\gamma}$ with $v_{0} \varphi=0$, hence $v_{0}$ induces a morphism $v: \mathcal{M} \rightarrow k\langle C\rangle_{\gamma}$. Furthermore, the section $V_{0} / V_{\infty} \hookrightarrow k\langle A\rangle$ which sends $a_{n m}^{l}+V_{\infty}$ to $e_{n m}^{l}$ determines another $\bar{T}_{1}$-controlled homomorphism $\tau_{0}: k\langle C\rangle_{\gamma} \rightarrow k\langle A\rangle_{\alpha}$ with $v_{0} \tau_{0}=1$, in particular if $\tau: k\langle C\rangle_{\gamma} \rightarrow \mathcal{M}$ is the morphism induced by $\tau_{0}$ we have that $v \tau=1$, hence $\mathcal{N} \simeq k\langle C\rangle_{\gamma} \oplus \mathcal{N}$ where $\mathcal{N}=$ Coker $\tau$. Notice that the morphism $\left(\varphi, \tau_{0}\right): k\langle B\rangle_{\beta} \oplus k\langle C\rangle_{\gamma} \rightarrow k\langle A\rangle_{\alpha}$ is a finite presentation of $\mathcal{N}$, and by (a) we have the following equality and inclusions for every $m \geq 1$

$$
k\langle A\rangle=\bigcap_{n \geq 1}\left[k\left\langle A_{n}\right\rangle+\varphi(k\langle B\rangle)\right] \oplus \tau(k\langle C\rangle) \subset k\left\langle A_{m}\right\rangle+\varphi(k\langle B\rangle)+\tau(k\langle C\rangle) \subset k\langle A\rangle,
$$

therefore $\lambda_{\mathcal{N}}=0$.

Observe that by (7.6) and (7.7) $k\langle C\rangle_{\gamma}$ is isomorphic to the free $\bar{T}_{1}$-controlled $k$-vector space which corresponds to $\mathcal{R}$ provided $\lambda_{\mathcal{M}}=\infty$, and to the direct sum of $\lambda_{\mathcal{M}}$ copies of $\mathcal{A}$ otherwise.

Lemma 7.19. Given a $f$. $p$. $\mathcal{R}$-module $\mathcal{N}$ with $\lambda_{\mathcal{M}}=0$, there exists another $f$. $p$. $\mathcal{R}$-module $\mathcal{N}$ with $\lambda_{\mathcal{N}}=\mu_{\mathcal{N}}=0$ such that $\mathcal{M} \simeq \mathcal{B}_{\mu_{\mathcal{M}}} \oplus \mathcal{N}$.

Proof. Suppose that $\mathcal{M}$ is the cokernel of $\varphi: k\langle B\rangle_{\beta} \rightarrow k\langle A\rangle_{\alpha}$ in $\mathbf{M}_{k}\left(\bar{T}_{1}\right)$. Since $\lambda_{\mathcal{M}}=0$ we have that $k\langle A\rangle=k\left\langle A_{m}\right\rangle+\varphi(k\langle B\rangle)$ for every $m \in \mathbb{N}$. Let $V_{\bullet}$ be the inverse system indexed by $\mathbb{N} \times \mathbb{N}$ given by

$$
V_{m n}=\frac{k\left\langle A_{m}\right\rangle}{k\left\langle A_{m}\right\rangle \cap \varphi\left(k\left\langle B_{n}\right\rangle\right)},
$$


and bonding homomorphisms induced by the obvious inclusions of vector spaces. There are inclusions $U_{m n}^{\varphi} \subset V_{m n}$ with quotients

$$
\frac{k\left\langle A_{m}\right\rangle}{k\left\langle A_{m}\right\rangle \cap \varphi(k\langle B\rangle)} \simeq \frac{k\left\langle A_{m}\right\rangle+\varphi(k\langle B\rangle)}{\varphi(k\langle B\rangle)}=\frac{k\langle A\rangle}{\varphi(k\langle B\rangle)} .
$$

This determines a short exact sequence in the pro-category of pro-vector spaces

$$
U_{\bullet}^{\varphi} \hookrightarrow V_{\bullet} \rightarrow \frac{k\langle A\rangle}{\varphi(k\langle B\rangle)} .
$$

Here we regard $k\langle A\rangle / \varphi(k\langle B\rangle)$ as the inverse system indexed by a singleton.

The vector space $U_{m n}^{\varphi}$ is always finite dimensional, because it is contained in $\varphi(k\langle B\rangle) / \varphi\left(k\left\langle B_{n}\right\rangle\right) \simeq \varphi\left(k\left\langle_{n-1} B\right\rangle\right)$ and ${ }_{n-1} B$ is a finite set. Since finite-dimensional vector spaces are artinian it is easy to see that $U_{\bullet}^{\varphi}$ satisfies the Mittag-Leffler property, in particular $\lim ^{1} U_{\bullet}^{\varphi}=0$ and hence by (5.B) $\operatorname{Ext}^{1}\left(k\langle A\rangle / \varphi(k\langle B\rangle), U_{\bullet}^{\varphi}\right)=$ 0 , so the sequence (a) admits a splitting $s: k\langle A\rangle / \varphi(k\langle B\rangle) \hookrightarrow V_{\bullet}$. This splitting is given by splittings $s_{m n}: k\langle A\rangle / \varphi(k\langle B\rangle) \hookrightarrow V_{m n}$ of the natural projections $V_{m n} \rightarrow$ $k\langle A\rangle / \varphi(k\langle B\rangle)$ which are compatible with the bonding homomorphisms of $V_{\bullet}$.

Let $\tilde{C}$ be a basis of $k\langle A\rangle / \varphi(k\langle B\rangle)$. This basis is either finite $\tilde{C}=\left\{b_{1}, \ldots b_{\mu_{\mathcal{M}}}\right\}$ if $\mu_{\mathcal{M}} \in \mathbb{N}_{0}$, or infinite countable $\tilde{C}=\left\{b_{n}\right\}_{n \in \mathbb{N}_{0}}$ if $\mu_{\mathcal{M}}=\infty$. Moreover, since $\varphi$ is controlled there exists an increasing sequence of natural numbers $\left\{l_{n}\right\}_{n>1}$ with $\varphi\left(k\left\langle B_{l_{n}}\right\rangle\right) \subset k\left\langle A_{n}\right\rangle$. We choose elements $b_{m}^{n-1} \in k\left\langle A_{n}\right\rangle$ and $y_{m}^{n-1} \in k\left\langle B_{l_{n}}\right\rangle$ such that $b_{m}^{n-1}+\varphi\left(k\left\langle B_{l_{n}}\right\rangle\right)=s_{n, l_{n}}\left(b_{m}\right) \in V_{n, l_{n}}=k\left\langle A_{n}\right\rangle / \varphi\left(k\left\langle B_{l_{n}}\right\rangle\right)$ and $\varphi\left(y_{m}^{n-1}\right)=$ $b_{m}^{n}-b_{m}^{n-1}$ for every $n \in \mathbb{N}$ and $m$ in the corresponding range. Furthermore, we define the sets ${ }^{n} C \subset k\left\langle A_{n+1}\right\rangle$ and $C$ in the following way: ${ }^{n} C=\left\{b_{1}^{n}, \ldots b_{\mu_{x}}^{n}\right\}$ and $C=\coprod_{n \in \mathbb{N}_{0}}{ }^{n} C$ if $\mu_{\mathcal{M}} \in \mathbb{N}_{0}$, and ${ }^{n} C=\left\{b_{0}^{n}, \ldots b_{n}^{n}\right\} \cup\left\{b_{m}^{m} ; m>n\right\}$ and $C=\bigcup_{n \in \mathbb{N}_{0}}{ }^{n} C=\left\{b_{m}^{n} ; n \geq m \geq 0\right\}$ if $\mu_{\mathcal{M}}=\infty$. Let $\gamma: C \rightarrow \mathbb{N}_{0} \subset[0,+\infty)$ be the height function given by $\gamma\left(b_{m}^{n}\right)=n$ and $\psi$ the endomorphism of $k\langle C\rangle_{\gamma}$ given by $\psi\left(b_{m}^{n}\right)=b_{m}^{n+1}-b_{m}^{n}$.

One readily checks that Coker $\psi=\mathfrak{i}(k\langle A\rangle / \varphi(k\langle B\rangle))$ and the natural projection $k\langle C\rangle_{\gamma} \rightarrow \mathfrak{i}(k\langle A\rangle / \varphi(k\langle B\rangle))$ is given by the homomorphism $p_{1}: k\langle C\rangle \rightarrow k\langle A\rangle / \varphi(k\langle B\rangle)=$ $k\langle\tilde{C}\rangle$ defined by $p_{1}\left(b_{m}^{n}\right)=b_{m}$. For this one uses the finite presentations constructed in the proof of (4.3) and, if $\mu_{\mathcal{M}}=\infty$, the bijection $\mathbb{N}_{0} \approx C$ which sends $m \in \mathbb{N}_{0}$, with $\frac{n(n-1)}{2} \leq m<\frac{(n+1) n}{2}$ for some $n \in \mathbb{N}_{0}$, to $b_{m-\frac{n(n-1)}{2}}^{n-1}$. Moreover, by (7.11) Coker $\psi=\mathcal{B}_{\mu_{\mathcal{M}}}$.

The homomorphism $\tau_{0}: k\langle C\rangle \rightarrow k\langle A\rangle$ induced by the inclusions ${ }^{n} C \subset k\left\langle A_{n+1}\right\rangle \subset$ $k\langle A\rangle$ determines a controlled homomorphism $\tau_{0}: k\langle C\rangle_{\gamma} \rightarrow k\langle A\rangle_{\alpha}$. Moreover, the homomorphism $\tau_{1}: k\langle C\rangle \rightarrow k\langle B\rangle$ given by $\tau_{1}\left(b_{m}^{n}\right)=y_{m}^{n}$ defines a controlled homomorphism $\tau_{1}: k\langle C\rangle_{\gamma} \rightarrow k\langle B\rangle_{\beta}$ with $\varphi \tau_{1}=\tau_{0} \psi$, hence $\tau_{0}$ gives rise to a $\mathbf{M}_{k}\left(\bar{T}_{1}\right)$ module morphism $\tau: \mathcal{B}_{\mu_{\mathcal{M}}} \rightarrow \mathcal{M}$.

Let us check that $\tau$ is a monomorphism. Given a free $\bar{T}_{1}$-controlled vector space $k\langle D\rangle_{\phi}$ Yoneda's lemma yields a natural identification $\operatorname{Hom}_{\mathcal{R}}\left(k\langle D\rangle_{\phi}, \mathfrak{i}(k\langle A\rangle / \varphi(k\langle B\rangle))\right)=$ $\operatorname{Hom}_{k}(k\langle D\rangle, k\langle A\rangle / \varphi(k\langle B\rangle))$. This identification carries a morphism $v: k\langle D\rangle_{\phi} \rightarrow$ $\mathfrak{i}(k\langle A\rangle / \varphi(k\langle B\rangle))$ represented by $v_{0}: k\langle D\rangle_{\phi} \rightarrow k\langle C\rangle_{\gamma}$ to the vector space homomorphism $p_{1} v_{0}$. If $p_{2}: k\langle A\rangle \rightarrow k\langle A\rangle / \varphi(k\langle B\rangle)$ is the natural projection then $p_{2} \varphi=0$ and $p_{1}=p_{2} \tau_{0}$. Moreover $\tau v=0$ if and only if $\tau_{0} v_{0}=\varphi \eta$ for some controlled homomorphism $\eta: k\langle D\rangle_{\phi} \rightarrow k\langle B\rangle_{\beta}$, so in this case $p_{1} v_{0}=p_{2} \tau_{0} v_{0}=p_{2} \varphi \eta=0$, it is, $v=0$, therefore $\tau$ is a monomorphism. 
By (7.12) if $\mathcal{N}=$ Coker $\tau$ then $\mathcal{M} \simeq \mathcal{B}_{\mu_{\mathcal{M}}} \oplus \mathcal{N}$. The morphism $\left(\varphi, \tau_{0}\right): k\langle B\rangle_{\beta} \oplus$ $k\langle C\rangle_{\gamma} \rightarrow k\langle A\rangle_{\alpha}$ is a finite presentation of $\mathcal{N}$ and by construction $\varphi(k\langle B\rangle)+$ $\tau_{0}(k\langle C\rangle)=k\langle A\rangle$, it is $\lambda_{\mathcal{N}}=\mu_{\mathcal{N}}=0$.

Lemma 7.20. Let $\mathcal{M}$ be a f. $p$. R-module with $\lambda_{\mathcal{M}}=\mu_{\mathcal{M}}=0$ then there exists another one $\mathcal{N}$ with $\lambda_{\mathcal{N}}=\mu_{\mathcal{N}}=\nu_{\mathcal{N}}=0$ such that $\mathcal{N} \simeq \mathcal{C}_{\nu_{\mathcal{M}}} \oplus \mathcal{N}$.

In the proof of this lemma we shall use the next one.

Lemma 7.21. Let $\left\{d_{n}\right\}_{n \in \mathbb{N}}$ be an increasing sequence of integers with $\lim _{n \rightarrow \infty} d_{n}=$ $\infty$. Consider the set $A=\left\{(n, m) ; n \in \mathbb{N}, m \leq d_{n}\right\} \subset \mathbb{N} \times \mathbb{N}$, the height function $\alpha: A \rightarrow \mathbb{N}_{0} \subset[0,+\infty)$ with $\alpha(n, m)=n$, and the endomorphism $\varphi$ of $k\langle A\rangle_{\alpha}$ with $\varphi(n, m)=(n, m)$ if $n=1$ or $n>1$ and $m>d_{n-1}$, and $\varphi(n, m)=(n, m)-(n-1, m)$ otherwise. Then Coker $\varphi$ is isomorphic to $\mathcal{C}_{\infty}$.

Proof. Consider the infinite countable subsets

$$
A_{1}=\left\{(1, m) ; 1 \leq m \leq d_{1}\right\} \cup\left\{(n, m) ; n>1, d_{n-1}<m \leq d_{n}\right\} \subset A,
$$

$A_{2}=A-A_{1}, B_{1}=\{(n+1) n / 2\}_{n \in \mathbb{N}_{0}} \subset \mathbb{N}_{0}$ and $B_{2}=\mathbb{N}_{0}-B_{1}$. The lexicographic order from the left on $A$ is a well order without limit elements, since the second coordinate of an element $(n, m) \in A$ is bounded by $d_{n}$, hence the restriction of this order to the subsets $A_{1}$ and $A_{2}$ induces enumerations $A_{1}=\left\{e_{1}^{n}\right\}_{n \in \mathbb{N}_{0}}, A_{2}=$ $\left\{e_{n}^{2}\right\}_{n \in \mathbb{N}_{0}}$. Similarly the usual order in $\mathbb{N}_{0}$ induces enumerations in the subsets $B_{1}=\left\{f_{1}^{n}\right\}_{n \in \mathbb{N}_{0}}$ and $B_{2}=\left\{f_{2}^{n}\right\}_{n \in \mathbb{N}_{0}}$. Now the theorem follows from the bijection $\mathbb{N}_{0} \approx A$ which sends $f_{i}^{n}$ to $e_{i}^{n}\left(i=1,2 ; n \in \mathbb{N}_{0}\right)$.

Proof of (7.20). If $\mathcal{M}=\operatorname{Coker}\left[\varphi: k\langle B\rangle_{\beta} \rightarrow k\langle A\rangle_{\alpha}\right]$ the equalities $\lambda_{\mathcal{M}}=\mu_{\mathcal{M}}=0$ are equivalent to $\varphi(k\langle B\rangle)=k\langle A\rangle$. Let $\phi: \lim U_{\bullet}^{\varphi} \rightarrow U_{\bullet}^{\varphi}$ be the canonical promorphism. This pro-morphism is given by vector space homomorphisms $\phi_{m n}: \lim U_{\bullet}^{\varphi} \rightarrow$ $U_{m n}^{\varphi}$ compatible with the bonding homomorphisms of $U_{\bullet}^{\varphi}$. Since $\varphi$ is controlled there is an increasing sequence of natural numbers $\left\{m_{n}\right\}_{n \geq 1}$ such that $\varphi\left(k\left\langle B_{m_{n}}\right\rangle\right) \subset$ $k\left\langle A_{n}\right\rangle$.

If $\nu_{\mathcal{M}} \in \mathbb{N}_{0}$ and $\left\{a_{1}, \ldots a_{\nu_{\mathcal{M}}}\right\}$ is a basis of $\lim U_{\bullet}^{\varphi}$ we define ${ }^{n} C=\left\{a_{1}^{n}, \ldots a_{\nu_{\mathcal{M}}}^{n}\right\} \subset$ $k\left\langle A_{n}\right\rangle$ as a set such that $\phi_{n, m_{n}}\left(a_{i}\right)=a_{i}^{n}+\varphi\left(k\left\langle B_{m_{n}}\right\rangle\right)\left(1 \leq i \leq \nu_{\mathcal{M}}\right)$, and choose elements $y_{i}^{n} \in k\left\langle B_{m_{n-1}}\right\rangle$ if $n>1$ and $y_{i}^{1} \in k\langle B\rangle$ with $a_{i}^{n}-a_{i}^{n-1}=\varphi\left(y_{i}^{n}\right)$ $(n>1)$ and $a_{i}^{1}=\varphi\left(y_{i}^{1}\right)$. If $\nu_{\mathcal{M}}=\infty$ we take ${ }^{n} C=\left\{a_{i}^{n}\right\}_{i=1}^{d_{n}} \subset k\left\langle A_{n}\right\rangle$ such that $\left\{a_{i}^{n}+\varphi\left(k\left\langle B_{m_{n}}\right\rangle\right)\right\}$ is a basis of $\phi_{n, m_{n}}\left(\lim U_{\bullet}^{\varphi}\right)$, here we use that $U_{\bullet}^{\varphi}$ is an inverse system of finite-dimensional vector spaces, compare the proof of (7.19). The bonding homomorphisms of $U_{\bullet}^{\varphi}$ induce surjections $\phi_{n+1, m_{n+1}}\left(\lim U_{\bullet}^{\varphi}\right) \rightarrow \phi_{n, m_{n}}\left(\lim U_{\bullet}^{\varphi}\right)$, hence $d_{n} \leq d_{n+1}$ and we can suppose without loose of generality that there exist $y_{i}^{n} \in k\left\langle B_{m_{n-1}}\right\rangle(n>1)$ and $y_{i}^{1} \in k\langle B\rangle$ such that $a_{i}^{n}-a_{i}^{n-1}=\varphi\left(y_{i}^{n}\right)$ if $n>1$ and $i \leq d_{n-1}$, and $a_{i}^{n}=\varphi\left(y_{i}^{n}\right)$ if $n>1$ and $d_{n-1}<i \leq d_{n}$ or $n=1$ and $i \leq d_{1}$.

We define the height function $\gamma: C=\coprod_{n>1}{ }^{n} C \rightarrow \mathbb{N}_{0} \subset[0,+\infty)$ as $\gamma\left(a_{i}^{n}\right)=$ $n$, and the controlled homomorphisms $\tau_{0}: k\langle C\rangle_{\gamma} \rightarrow k\langle A\rangle_{\alpha}, \tau_{1}: k\langle C\rangle_{\gamma} \rightarrow k\langle B\rangle_{\beta}$, $\psi: k\langle C\rangle_{\gamma} \rightarrow k\langle C\rangle_{\gamma}$ by $\tau_{0}\left(a_{i}^{n}\right)=a_{i}^{n}, \tau_{1}\left(a_{i}^{n}\right)=y_{i}^{n}$, and $\psi\left(a_{i}^{n}\right)=a_{i}^{n}-a_{i}^{n-1}$ if $n>1$, and $\nu_{\mathcal{M}} \in \mathbb{N}_{0}$ or $\nu_{\mathcal{M}}=\infty$ and $i \leq d_{n-1}$, and $\psi\left(a_{i}^{n}\right)=a_{i}^{n}$ otherwise. One can readily check, by using the bijection $\mathbb{N} \approx \mathbb{N}_{0}: n \mapsto n-1$ if $\nu_{\mathcal{M}} \in \mathbb{N}_{0}$ or (7.21) if $\nu_{\mathcal{M}}=\infty$, that Coker $\psi \simeq \mathcal{C}_{\nu_{\mathcal{M}}}$. Moreover, $\left(\tau_{1}, \tau_{0}\right): \psi \rightarrow \varphi$ is a morphism in $\operatorname{pair}\left(\mathbf{M}_{k}\left(\bar{T}_{1}\right)\right)$ which induces an $\mathcal{R}$-module morphism $\tau: \mathcal{C}_{\nu_{\mathcal{M}}} \rightarrow \mathcal{M}$.

In order to check that $\tau$ is a monomorphism of $\mathbf{M}_{k}\left(\bar{T}_{1}\right)$-modules we are going to prove that $\phi$ is a monomorphism of pro-vector spaces if $\nu_{\mathcal{M}} \in \mathbb{N}_{0}$. In this case Ker $\phi$ 
is an inverse system of finite-dimensional vector spaces, in particular it satisfies the Mittag-Leffler property. If we apply the left-exact functor lim to the exact sequence of pro-vector spaces

$$
\operatorname{Ker} \phi \hookrightarrow \lim U_{\bullet}^{\varphi} \stackrel{\phi}{\longrightarrow} U_{\bullet}^{\varphi}
$$

we get another one

$$
\lim \operatorname{Ker} \phi \hookrightarrow \lim \lim U_{\bullet}^{\varphi} \stackrel{=}{\longrightarrow} \lim U_{\bullet}^{\varphi},
$$

so $\lim \operatorname{Ker} \phi=0$ and hence $\operatorname{Ker} \phi=0$ by [11] II.6.2 Lemma 2, therefore $\phi$ is a monomorphism. In particular there exists $N \in \mathbb{N}$ big enough such that $\phi_{n, m_{n}}$ is an injective homomorphism for every $n \geq N$. We set $N=1$ if $\nu_{\mathcal{M}}=\infty$. Now it is easy to see that the injection $\psi_{n}: k\left\langle C_{n+1}\right\rangle \hookrightarrow k\left\langle C_{n}\right\rangle$ given by the restriction of $\psi$ is the kernel of the next composition whenever $n \geq N$

$$
k\left\langle C_{n}\right\rangle \stackrel{\tau_{0}}{\rightarrow} k\left\langle A_{n}\right\rangle \rightarrow \frac{k\left\langle A_{n}\right\rangle}{\varphi\left(k\left\langle B_{m_{n}}\right\rangle\right)}=U_{n, m_{n}}^{\varphi} .
$$

Any morphism $v: k\langle D\rangle_{\chi} \rightarrow \mathcal{C}_{\nu_{\mathcal{M}}}$ is represented by a controlled homomorphism $v_{0}: k\langle D\rangle_{\chi} \rightarrow k\langle C\rangle_{\gamma}$. Suppose that $\tau v=0$. This means that there exists another controlled homomorphism $\eta: k\langle D\rangle_{\chi} \rightarrow k\langle B\rangle_{\beta}$ with $\tau_{0} v_{0}=\varphi \eta$. By the alternative characterization of controlled homomorphisms given in Subsection 3.1 we see that there exists an increasing sequence of natural numbers $\left\{p_{n}\right\}_{n \geq 1}$ such that $v_{0}\left(k\left\langle D_{p_{n}}\right\rangle\right) \subset k\left\langle C_{n}\right\rangle$ and $\eta\left(k\left\langle D_{p_{n}}\right\rangle\right) \subset k\left\langle B_{m_{n}}\right\rangle$, hence if $n \geq N$ then there exists a unique homomorphism $\sigma_{n}: k\left\langle D_{p_{n}}\right\rangle \rightarrow k\left\langle C_{n+1}\right\rangle$ such that $\psi_{n} \sigma_{n}: k\left\langle D_{p_{n}}\right\rangle \rightarrow k\left\langle C_{n}\right\rangle$ is the restriction of $v_{0}$. If $\sigma^{\prime}: k\left\langle_{p_{N}-1} D\right\rangle \rightarrow k\langle C\rangle$ is any homomorphism such that $\psi \sigma^{\prime}$ coincides with the restriction of $v_{0}$ to $k\left\langle_{p_{N}-1} D\right\rangle$ we define the controlled homomorphism $\sigma: k\langle D\rangle_{\chi} \rightarrow k\langle C\rangle_{\gamma}$ by $\sigma(d)=\sigma_{n}(d)$ if $d \in p_{p_{n+1}-1} D_{p_{n}}(n \geq N)$ and $\sigma(d)=\sigma^{\prime}(d)$ if $d \in p_{N-1} D$. This controlled homomorphism satisfies $\psi \sigma=v_{0}$ therefore $v=0$ and hence $\tau$ is a monomorphism.

Since $\tau$ is a monomorphism if we define $\mathcal{N}=$ Coker $\tau$ we get by (7.17) that $\mathcal{M} \simeq \mathcal{C}_{\nu_{\mathcal{M}}} \oplus \mathcal{N}$. Now one can check that $\lambda_{\mathcal{N}}=\mu_{\mathcal{N}}=\nu_{\mathcal{N}}=0$ by using that $\mathcal{N}=\operatorname{Coker}\left[\left(\varphi, \tau_{0}\right): k\langle B\rangle_{\beta} \oplus k\langle C\rangle_{\gamma} \rightarrow k\langle A\rangle_{\alpha}\right]$.

Lemma 7.22. If $\mathcal{M}$ is a f. $p$. $\mathcal{R}$-module with $\lambda_{\mathcal{M}}=\mu_{\mathcal{M}}=\nu_{\mathcal{M}}=0$ then $\mathcal{M}=0$.

Proof. If $\mathcal{M}=\operatorname{Coker}\left[\varphi: k\langle B\rangle_{\beta} \rightarrow k\langle A\rangle_{\alpha}\right]$ the conditions of the statement are equivalent to $\varphi(k\langle B\rangle)=k\langle A\rangle$ and $\lim U_{\bullet}^{\varphi}=0$. In the proof of (7.19) we checked that $U_{\bullet}^{\varphi}$ satisfies the Mittag-Leffler property, hence $U_{\bullet}^{\varphi}=0$ is a trivial pro-vector space by [11] II.6.2 Lemma 2. This means that if $\left\{m_{n}\right\}_{n>1}$ is an increasing sequence such that $\varphi\left(k\left\langle B_{m_{n}}\right\rangle\right) \subset k\left\langle A_{n}\right\rangle$ (see Subsection 3.1) then there exists another increasing sequence $\left\{p_{n}\right\}_{n \geq 1}$ such that the next bonding homomorphisms are trivial

$$
U_{p_{n+1}, m_{p_{n+1}}}^{\varphi}=\frac{k\left\langle A_{p_{n+1}}\right\rangle}{\varphi\left(k\left\langle B_{m_{p_{n}+1}}\right\rangle\right)} \stackrel{0}{\longrightarrow} \frac{k\left\langle A_{p_{n}}\right\rangle}{\varphi\left(k\left\langle B_{m_{p_{n}}}\right\rangle\right)}=U_{p_{n}, m_{p_{n}}}^{\varphi} .
$$

It is, $k\left\langle A_{p_{n+1}}\right\rangle \subset \varphi\left(k\left\langle B_{m_{p_{n}}}\right\rangle\right)$. Hence we can define a controlled homomorphism $\psi: k\langle A\rangle_{\alpha} \rightarrow k\langle B\rangle_{\beta}$ sending $a \in p_{p_{n+2}-1} A_{p_{n+1}}(n \geq 1)$ to any element $b \in B_{m_{p_{n}}}$ such that $\varphi(b)=a$, and if $a \in{ }_{p_{2}} A$ we take any $\psi(a)=b \in k\langle B\rangle$ such that $\varphi(b)=a$. This morphism satisfies $\varphi \psi=1$ hence $\varphi$ is an epimorphism and $\mathcal{M}=\operatorname{Coker} \varphi=0$. 


\section{RePRESENTATIONS OF THE $n$-SUBSPACE QUIVER}

The $n$-subspace quiver $Q_{n}$ is the next directed graph

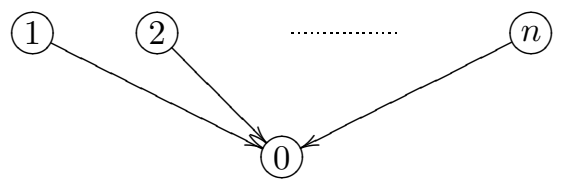

Fixed any field $k$, a representation $\underline{V}$ of $Q_{n}$ is a diagram of $k$-vector spaces indexed by $Q_{n}$, it is, $n+1$ vector spaces $V_{0}, V_{1}, \ldots V_{n}$ together with homomorphisms $V_{i} \rightarrow V_{0}(1 \leq i \leq n)$. Morphisms of representations are commutative diagrams. The category $\operatorname{rep}_{Q_{n}}$ of representations of $Q_{n}$ is an abelian category. It is equivalent to the category of $k Q_{n}$-modules, where $k Q_{n}$ is the path algebra of $Q_{n}$, whose dimension is $\operatorname{dim} k Q_{n}=2 n+1$. A representation is said to be finite-dimensional provided $V_{i}$ is a finite-dimensional vector space for every $0 \leq i \leq n$. Finitely presented (or equivalently finite-dimensional) $k Q_{n}$-modules correspond to finitedimensional representations under this equivalence and indecomposable representations are finite-dimensional, hence by the classical Krull-Schmidt theorem the monoid Iso $\left(\mathbf{r e p}_{Q_{n}}^{\mathrm{fin}}\right)$ of isomorphisms classes of finite-dimensional representations of $Q_{n}$ is the free abelian monoid generated by the (isomorphism classes of) indecomposable representations. The representation type of the quiver $Q_{n}$ is that of its path algebra.

An $n$-subspace $\underline{V}$ is a representation of $Q_{n}$ such that the homomorphisms $V_{i} \rightarrow V_{0}$ are inclusions of subspaces $V_{i} \subset V_{0}(1 \leq i \leq n)$. The category $\mathbf{s u b}_{n}$ (resp. sub $\mathbf{s u b}_{n}^{\text {fin }}$ ) of (finite-dimensional) $n$-subspaces is a full additive subcategory of $\operatorname{rep}_{Q_{n}}$ (resp. $\operatorname{rep}_{Q_{n}}^{\text {fin }}$ ). In fact direct summands in $\mathbf{r e p}_{Q_{n}}$ of $n$-subspaces are also $n$-subspaces, hence

Proposition 8.1. Iso $\left(\mathbf{s u b}_{n}^{\mathrm{fin}}\right)$ is the free abelian monoid generated by the isomorphism classes of indecomposable $n$-subspaces.

Up to isomorphism there are just $n$ indecomposable representations of $Q_{n}$ which are not $n$-subspaces, namely those with $V_{i}=k$ for some $1 \leq i \leq n$ and $V_{j}=0$ if $j \neq i$, therefore

Proposition 8.2. The representation type of $\mathbf{s u b}_{n}^{\mathrm{fin}}$ is the same as the $n$-subspace quiver.

We say that an $n$-subspace $\underline{V}$ is rigid provided $V_{i} \subset \sum_{j \neq 0, i} V_{j}(1 \leq i \leq n)$ and $V_{0}=\sum_{i=1}^{n} V_{i}$. As before, the category $\mathbf{s u b}_{n}^{\text {rig }}\left(\right.$ resp. $\mathbf{s u b}_{n}^{\mathrm{fr}}$ ) of (finite-dimensional) rigid $n$-subspaces is an additive (small) subcategory of $\mathbf{s u b}_{n}$ (resp. $\mathbf{s u b}_{n}^{\mathrm{fin}}$ ) and direct summands of rigid $n$-subspaces are also rigid, so

Proposition 8.3. Iso( $\left(\mathbf{s u b}_{n}^{\mathrm{fr}}\right)$ is the free abelian monoid generated by the isomorphism classes of indecomposable rigid n-subspaces.

There is an additive "rigidification" functor

$$
\operatorname{sub}_{n} \longrightarrow \operatorname{sub}_{n}^{\text {rig }}: \underline{V} \mapsto \underline{V}^{\text {rig }}
$$

given by $V_{i}^{\mathrm{rig}}=V_{i} \cap\left(\sum_{j \neq 0, i} V_{j}\right)(1 \leq i \leq n)$ and $V_{0}^{\mathrm{rig}}=\sum_{i=1}^{n} V_{i}^{\mathrm{rig}}$, which is rightadjoint to the inclusion $\mathbf{s u b}_{n}^{\text {rig }} \subset \mathbf{s u b}_{n}$ and preserves finite-dimensional objects. 
The unit of this adjunction is the obvious natural inclusion $\underline{V}^{\text {rig }} \subset \underline{V}$, which is an equality if and only if $\underline{V}$ is already rigid.

In order to determine indecomposable rigid $n$-subspaces we consider the full inclusions of additive categories $\mathbb{F}^{i}: \mathbf{s u b}_{1} \rightarrow \operatorname{sub}_{n}(1 \leq i \leq n)$ sending a 1 -subspace $\underline{W}$ to the $n$-subspace $\mathbb{F}^{i} \underline{W}={ }^{i} \underline{W}$ with ${ }^{i} W_{0}=W_{0},{ }^{i} W_{i}=W_{1}$ and ${ }^{i} W_{j}=0$ otherwise.

Proposition 8.4. The natural inclusion $\underline{V}^{\text {rig }} \subset \underline{V}$ admits a (not natural) retraction in $\mathbf{s u b}_{n}$. More precisely, there exist 1 -subspaces $\underline{V}^{i}(1 \leq i \leq n)$ and an isomorphism $\underline{V} \simeq\left(\bigoplus_{i=1}^{n} \mathbb{F}^{i} \underline{V}^{i}\right) \oplus \underline{V}^{\text {rig }}$ such that the natural inclusion $\underline{V}^{\text {rig }} \subset \underline{V}$ corresponds to the inclusion of the direct summand.

Proof. By using the definition of $\underline{V}^{\text {rig }}$ we see that there is a short exact sequence of vector spaces

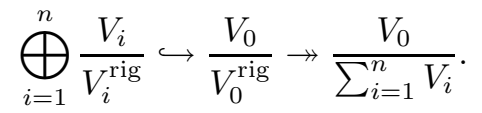

We define the 1-subspaces $\underline{V}^{i}(1 \leq i \leq n)$ as $V_{0}^{1}=\frac{V_{0}}{\sum_{i=1}^{n} V_{i}} \oplus \frac{V_{1}}{V_{1}^{\text {rig }}}, V_{1}^{1}=\frac{V_{1}}{V_{1}^{\text {rig }}}$ and $V_{0}^{i}=V_{1}^{i}=\frac{V_{i}}{V_{i}^{\text {rig }}}$ if $1<i \leq n$. Now the isomorphism of the statement follows from the former exact sequence.

By this proposition an indecomposable $n$-subspace is rigid unless it is isomorphic to $\mathbb{F}^{i} \underline{V}$ for some indecomposable 1 -subspace $\underline{V}$ and $1 \leq i \leq n$. It is known that such $\underline{V}$ must be either $k \rightarrow k$ or $0 \rightarrow k$, so there are just $2 n$ indecomposable $n$-subspaces which are not rigid, and $3 n$ indecomposable representations of $Q_{n}$ which are not rigid $n$-subspaces, in particular

Proposition 8.5. The category $\mathbf{s u b}_{n}^{\text {fr }}$ has the same representation type as the $n$ subspace quiver.

Remark 8.6. The representation type of the $n$-subspace quiver is well-known. It is finite for $n<4$, tame for $n=4$ and wild if $n>4$, see [8] and [14].

In [8] the finite sets of indecomposable representations of $Q_{n}$ are described for $n<4$ hence discarding the $3 n$ indecomposable representations previously described which are not rigid $n$-subspaces we get the next result.

Proposition 8.7. The following are complete lists of (representatives of the isomorphism classes of) indecomposable rigid $n$-subspaces for $n<4$

$$
\begin{aligned}
& \text { - } n=1 \text {, none, } \\
& \text { - } n=2, \underline{V}^{(2,1)}=(k \rightarrow k \leftarrow k) \text {, } \\
& \text { - } n=3 \text {, }
\end{aligned}
$$

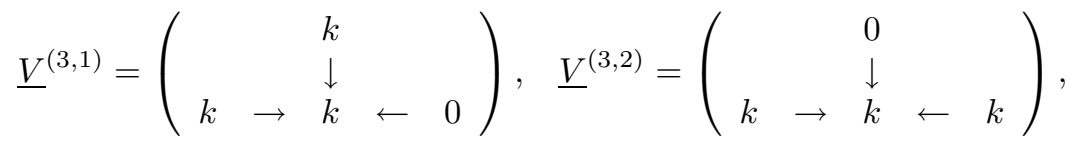

$$
\begin{aligned}
& \underline{V}^{(3,3)}=\left(\begin{array}{rrrrr} 
& k & & \\
& & \downarrow & \\
0 & \rightarrow & k & \leftarrow &
\end{array}\right), \quad \underline{V}^{(3,4)}=\left(\begin{array}{lllll} 
& & k & \\
& & \downarrow & \\
k & \rightarrow & k & \leftarrow \quad k
\end{array}\right),
\end{aligned}
$$

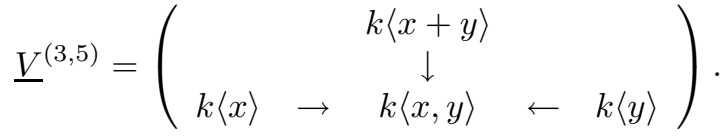


Remark 8.8. In [14] there is a (not finite) list of indecomposable representations of $Q_{4}$. We do not include the list here because it is quite tedious to describe, however the interested reader can easily find and remove the 12 indecomposable representations of $Q_{4}$ which are not rigid 4-subspaces, obtaining in this way a complete list of indecomposable rigid 4 -subspaces .

\section{Finitely PRESEnted $k(\mathbf{n})$-MOdUles AND Finite-Dimensional $n$-SUbSPACES}

Given an $n$-subspace $\underline{V}$ we define $\mathbb{M} \underline{V}: \mathbf{M}_{k}\left(\bar{T}_{n}\right)^{o p} \rightarrow \mathbf{A b}$ as the additive functor which sends an object $k\langle A\rangle_{\alpha}$ to the vector subspace $\mathbb{M} \underline{V}\left(k\langle A\rangle_{\alpha}\right) \subset \operatorname{Hom}_{k}\left(k\langle A\rangle, V_{0}\right)$ formed by the homomorphisms $\phi: k\langle A\rangle \rightarrow V_{0}$ such that there exists $M \geq 1$ depending on $\phi$ satisfying $\phi\left(A_{M}^{i}\right) \subset V_{i}(1 \leq i \leq n)$. This construction defines an exact full inclusion of additive categories

$$
\mathbb{M}: \operatorname{sub}_{n} \rightarrow \bmod \left(\mathbf{M}_{k}\left(\bar{T}_{n}\right)\right) .
$$

Proposition 9.1. If $\underline{\underline{V}}$ is a finite-dimensional n-subspace then the $\mathbf{M}_{k}\left(\bar{T}_{n}\right)$-module $\mathbb{M} \underline{\underline{V}}$ is finitely presented.

Proof. Let $\left\{w_{1}, \ldots w_{d}\right\}$ be a basis of $V_{0},\left\{w_{1}^{i}, \ldots w_{d_{i}}^{i}\right\}$ a basis of $V_{i}(1 \leq i \leq n)$, and $\phi_{i}: V_{i} \rightarrow V_{0}$ the inclusion. We define the sets $D=\left\{{ }_{m} w_{1}^{i}, \ldots m w_{d_{i}}^{i} ; 1 \leq i \leq n, m \geq 1\right\}$ and $C=D \sqcup\left\{w_{1}, \ldots w_{d}\right\}$, and the height functions $\gamma: C \rightarrow T_{n}^{0}$ and $\delta: D \rightarrow T_{n}^{0}$ with $\gamma\left(w_{j}\right)=v_{0}(1 \leq j \leq d)$ and $\gamma\left({ }_{m} w_{j}^{i}\right)=\delta\left({ }_{m} w_{j}^{i}\right)=v_{m}^{i}(1 \leq i \leq n, 1 \leq$ $\left.j \leq d_{i}, m \geq 1\right)$. Let $\rho: k\langle D\rangle_{\delta} \rightarrow k\langle C\rangle_{\gamma}$ be the controlled homomorphism defined as $\rho\left({ }_{m} w_{j}^{i}\right)={ }_{m} w_{j}^{i}-{ }_{m-1} w_{j}^{i}$ if $m>1$ and $\rho\left({ }_{1} w_{j}^{i}\right)={ }_{1} w_{j}^{i}-\phi_{i}\left(w_{j}^{i}\right)$ otherwise, and $p: k\langle C\rangle_{\gamma} \rightarrow \mathbb{M} \underline{V}$ the $\mathbf{M}_{k}\left(\bar{T}_{n}\right)$-module morphism determined by the $k$-vector space homomorphism $p_{0}: k\langle C\rangle \rightarrow V_{0}$ with $p_{0}\left(w_{j}\right)=w_{j}$ and $p_{0}\left(m w_{j}^{i}\right)=\phi_{i}\left(w_{j}^{i}\right)$. Here we use that $\operatorname{Hom}\left(k\langle C\rangle_{\gamma}, \mathbb{M} \underline{V}\right)=\mathbb{M} \underline{V}\left(k\langle C\rangle_{\gamma}\right)$ by Yoneda's lemma. Now it is immediate to check that $\mathbb{M} \underline{V}=$ Coker $\rho$ and $p$ is the natural projection.

One can check by using the finite presentation constructed in the proof of the former proposition that

Corollary 9.2. If $\underline{V}$ is a finite-dimensional rigid n-subspace then $\Phi_{n}([\mathbb{M} \underline{\underline{V}}])=0$.

By (9.1) the additive functor in (9.A) restricts to a functor $\mathbb{M}: \mathbf{s u b}_{n}^{\text {fin }} \rightarrow \mathbf{f p}\left(\mathbf{M}_{k}\left(\bar{T}_{n}\right)\right)$. Now we are going to construct a functor in the opposite direction. For this if $\varphi: k\langle B\rangle_{\beta} \rightarrow k\langle A\rangle_{\alpha}$ is a morphism in $\mathbf{M}_{k}\left(\bar{T}_{n}\right)$ we define the $k$-vector spaces $(1 \leq i \leq n)$

$$
W_{i}^{\varphi}=\frac{\bigcap_{m \geq 1}\left\{\left[k\left\langle A_{m}^{i}\right\rangle+\varphi(k\langle B\rangle)\right] \cap\left[\sum_{j \neq i} k\left\langle A_{m}^{j}\right\rangle+\varphi(k\langle B\rangle)\right]\right\}}{\varphi(k\langle B\rangle)} .
$$

Proposition 9.3. The vector space $W_{i}^{\varphi}$ is finite-dimensional $(1 \leq i \leq n)$.

This proposition is an immediate consequence of the next

Lemma 9.4. For any $m \geq 1$

$$
\operatorname{dim} \frac{\left[k\left\langle A_{m}^{i}\right\rangle+\varphi(k\langle B\rangle)\right] \cap\left[\sum_{j \neq i} k\left\langle A_{m}^{j}\right\rangle+\varphi(k\langle B\rangle)\right]}{\varphi(k\langle B\rangle)}<\aleph_{0} .
$$


Proof. One readily checks that

$$
\begin{gathered}
{\left[k\left\langle A_{m}^{i}\right\rangle+\varphi(k\langle B\rangle)\right] \cap\left[\sum_{j \neq i} k\left\langle A_{m}^{j}\right\rangle+\varphi(k\langle B\rangle)\right]=} \\
k\left\langle A_{m}^{i}\right\rangle \cap\left[\sum_{j \neq i} k\left\langle A_{m}^{j}\right\rangle+\varphi(k\langle B\rangle)\right]+\varphi(k\langle B\rangle),
\end{gathered}
$$

therefore

$$
\begin{gathered}
\frac{\left[k\left\langle A_{m}^{i}\right\rangle+\varphi(k\langle B\rangle)\right] \cap\left[\sum_{j \neq i} k\left\langle A_{m}^{j}\right\rangle+\varphi(k\langle B\rangle)\right]}{\varphi(k\langle B\rangle)} \simeq \\
\frac{k\left\langle A_{m}^{i}\right\rangle \cap\left[\sum_{j \neq i} k\left\langle A_{m}^{j}\right\rangle+\varphi(k\langle B\rangle)\right]}{k\left\langle A_{m}^{i}\right\rangle \cap \varphi(k\langle B\rangle)} .
\end{gathered}
$$

Since $\varphi$ is a controlled homomorphism there exists $M \geq 1$ such that $\varphi\left(B_{M}^{i}\right) \subset$ $k\left\langle A_{m}^{i}\right\rangle(1 \leq i \leq n)$, hence

$$
\sum_{j \neq i} k\left\langle A_{m}^{j}\right\rangle+\varphi(k\langle B\rangle)=\sum_{j \neq i} k\left\langle A_{m}^{j}\right\rangle+\varphi\left(k\left\langle_{M-1} B\right\rangle\right)+\varphi\left(k\left\langle B_{M}^{i}\right\rangle\right) .
$$

The set ${ }_{M-1} B$ is finite, hence there exists $N \geq 0$ big enough with $\varphi\left({ }_{M-1} B\right) \subset$ $k\left\langle_{N} A\right\rangle$. Let us check that the next homomorphism induced by the inclusion ${ }_{N} A_{m}^{i} \subset$ $A_{m}^{i}$ is an isomorphism

$$
\text { (c) } \frac{\left.k{ }_{N} A_{m}^{i}\right\rangle \cap\left[\sum_{j \neq i} k\left\langle A_{m}^{j}\right\rangle+\varphi(k\langle B\rangle)\right]}{k\left\langle_{N} A_{m}^{i}\right\rangle \cap \varphi(k\langle B\rangle)} \rightarrow \frac{k\left\langle A_{m}^{i}\right\rangle \cap\left[\sum_{j \neq i} k\left\langle A_{m}^{j}\right\rangle+\varphi(k\langle B\rangle)\right]}{k\left\langle A_{m}^{i}\right\rangle \cap \varphi(k\langle B\rangle)} \text {. }
$$

The injectivity is obvious. Now by (b) an arbitrary element in the range of (c) is represented by an element $a_{i} \in k\left\langle A_{m}^{i}\right\rangle$ such that there are $a_{j} \in k\left\langle A_{m}^{j}\right\rangle(j \neq i)$, $a_{i}^{\prime} \in k\left\langle A_{m}^{i}\right\rangle \cap \varphi(k\langle B\rangle), b \in \varphi\left(k\left\langle_{M-1} B\right\rangle\right)$, and $b_{i} \in \varphi\left(k\left\langle B_{M}^{i}\right\rangle\right)$ such that

$$
\sum_{j \neq i} a_{j}+b+b_{i}=a_{i}+a_{i}^{\prime}
$$

but $a_{i}+a_{i}^{\prime}-b_{i} \in k\left\langle A_{m}^{i}\right\rangle,\left(\oplus_{j=1}^{n} k\left\langle A_{m}^{j}\right\rangle\right) \cap k\left\langle_{N} A\right\rangle=\oplus_{j=1}^{n} k\left\langle_{N} A_{m}^{j}\right\rangle$, and $\sum_{j \neq i} a_{j}-$ $\left(a_{i}+a_{i}^{\prime}-b_{i}\right)=b \in k\left\langle_{N} A\right\rangle$, therefore $a_{j}, a_{i}+a_{i}^{\prime}-b_{i} \in k\left\langle_{N} A\right\rangle(j \neq i)$, and $a_{i}^{\prime}-b_{i} \in k\left\langle A_{m}^{i}\right\rangle \cap \varphi\langle B\rangle$, so $a_{i}+a_{i}^{\prime}-b_{i}$ represents the same element as $a_{i}$ in the range of (c), hence the homomorphism (c) is surjective. Now the proposition follows from the isomorphisms (a) and (c), and the finiteness of the set ${ }_{N} A_{m}^{i}$.

By (9.3) the vector space $W_{0}^{\varphi}=\sum_{i=1}^{n} W_{i}^{\varphi}$ together with the subspaces $W_{1}^{\varphi}, \ldots W_{n}^{\varphi}$ define a finite dimensional $n$-subspace $\underline{W}^{\varphi}$.

Proposition 9.5. There is an additive functor $\mathbb{S}: \mathbf{f p}\left(\mathbf{M}_{k}\left(\bar{T}_{n}\right)\right) \rightarrow \mathbf{s u b}_{n}^{\text {fin }}$ which sends $\mathcal{M}=\operatorname{Coker} \varphi$ to $\mathbb{S} \mathcal{M}=\underline{W}^{\varphi}$.

Proof. By using the alternative description of controlled homomorphisms in $\mathbf{M}_{k}\left(\bar{T}_{n}\right)$ given in Subsection 3.1 one can easily check hat the correspondence $\varphi \mapsto \underline{W}^{\varphi}$ is a functor from pair $\left(\mathbf{M}_{k}\left(\bar{T}_{n}\right)\right)$ to the category of $n$-subspaces. Furthermore, this functor factors through the natural equivalence relation $\sim$, therefore the proposition follows by $(2.1)$. 
The functors $\mathbb{S}$ and $\mathbb{M}$ are not adjoint. Moreover, one readily checks that

Proposition 9.6. The $n$-subspace $\mathbb{S} \mathcal{M}$ is rigid for every $f . p . \mathbf{M}_{k}\left(\bar{T}_{n}\right)$-module $\mathcal{M}$. Moreover, given a finite-dimensional $n$-subspace $\underline{V}$ there is a natural isomorphism $\mathbb{S M} \underline{V} \simeq \underline{V}^{\text {rig }}$

For the second statement of the former proposition one uses the finite presentations constructed in the proof of (9.1).

Corollary 9.7. The image of the functor $\mathbb{S}$ is the category of finite-dimensional rigid $n$-subspaces.

Corollary 9.8. For every f. p. $\mathbf{M}_{k}\left(\bar{T}_{n}\right)$-module $\mathcal{M}$ there is a natural isomorphism SMSM $\simeq \mathbb{S} \mathcal{M}$.

As we pointed out in the introduction a key step to obtain a presentation of Iso $\left(\mathbf{f p}\left(\mathbf{M}_{k}\left(\bar{T}_{n}\right)\right)\right)$ is relating the decomposition problem in $\mathbf{f p}\left(\mathbf{M}_{k}\left(\bar{T}_{n}\right)\right)$ to the decomposition problem in $\mathbf{f} \mathbf{p}\left(\mathbf{M}_{k}\left(\bar{T}_{1}\right)\right)$ and $\mathbf{s u b} \mathbf{b}_{n}^{\text {fin }}$. This is what we do in the next two propositions.

Proposition 9.9. For any f. p. $\mathbf{M}_{k}\left(\bar{T}_{n}\right)$-module $\mathcal{M}$ there exists another one $\mathcal{N}$ with $\mathbb{S} \mathcal{N}=0$ such that $\mathcal{M} \simeq \mathcal{N} \oplus \mathbb{M} \mathbb{S} \mathcal{M}$.

Proof. Suppose that $\mathcal{M}$ is the cokernel of $\varphi: k\langle B\rangle_{\beta} \rightarrow k\langle A\rangle_{\alpha}$ in $\mathbf{M}_{k}\left(\bar{T}_{n}\right)$. By the alternative description of controlled homomorphisms given in Subsection 3.1 we can choose an increasing sequence of natural numbers $\left\{M_{m}\right\}_{m \geq 1}$ with $\varphi\left(B_{M_{m}}^{i}\right) \subset$ $k\left\langle A_{m}^{i}\right\rangle(1 \leq i \leq n)$. We define the inverse systems of vector spaces $X_{\bullet}^{i}, Y_{\bullet}^{i}, Z_{\bullet}^{i}$ $(1 \leq i \leq n)$ indexed by $\mathbb{N}$ in the following way

$$
\begin{gathered}
X_{m}^{i}=\frac{\varphi(k\langle B\rangle)}{\varphi\left(k\left\langle B_{M_{m}}^{i}\right\rangle\right)}, \\
Y_{m}^{i}=\frac{\left[k\left\langle A_{m}^{i}\right\rangle+\varphi(k\langle B\rangle)\right] \cap\left[\sum_{j \neq i} k\left\langle A_{m}^{j}\right\rangle+\varphi(k\langle B\rangle)\right]}{\varphi\left(k\left\langle B_{M_{m}}^{i}\right\rangle\right)}, \\
Z_{m}^{i}=\frac{\left[k\left\langle A_{m}^{i}\right\rangle+\varphi(k\langle B\rangle)\right] \cap\left[\sum_{j \neq i} k\left\langle A_{m}^{j}\right\rangle+\varphi(k\langle B\rangle)\right]}{\varphi(k\langle B\rangle)} .
\end{gathered}
$$

The bonding homomorphisms are induced by the obvious inclusions of vector spaces. The short exact sequences $X_{m}^{i} \hookrightarrow Y_{m}^{i} \stackrel{p_{i}^{m}}{\rightarrow} Z_{m}^{i}$ are compatible with the bonding homomorphisms, so they give rise to short exact sequences $X_{\bullet}^{i} \hookrightarrow Y_{\bullet}^{i} \stackrel{p_{i}}{\rightarrow} Z_{\bullet}^{i}$ in the abelian pro-category of pro-vector spaces. Moreover, $\lim Z_{\bullet}^{i}=\cap_{m \geq 1} Z_{m}^{i}=W_{i}^{\varphi}$. Let $\psi_{i}: W_{i}^{\varphi} \rightarrow Z_{\bullet}^{i}$ be the canonical pro-morphism, which is induced by the inclusions $W_{i}^{\varphi} \subset Z_{m}^{i}$. Here we regard $W_{i}^{\varphi}$ as an inverse system indexed by a singleton.

The bonding homomorphisms of the inverse system $X_{\bullet}^{i}$ are surjective, therefore $\lim ^{1} X_{\bullet}^{i}=0$, and by (5.B) $\operatorname{Ext}^{1}\left(W_{i}^{\varphi}, X_{\bullet}^{i}\right)=0$, so there exists a pro-morphism $\tilde{\psi}_{i}$ such that the next diagram commutes

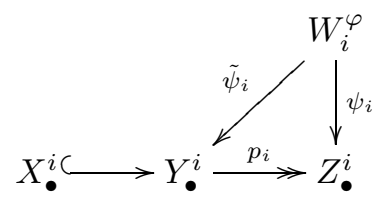


The pro-morphism $\tilde{\psi}_{i}$ is represented by a sequence of homomorphisms $\tilde{\psi}_{i}^{m}: W_{i}^{\varphi} \rightarrow$ $Y_{m}^{i}(m \geq 1)$ which are compatible with the bonding homomorphisms of $Y_{\bullet}^{i}$, and such that the composition $p_{i}^{m} \tilde{\psi}_{i}^{m}: W_{i}^{\varphi} \subset Z_{m}^{i}$ is the inclusion.

If $\left\{a_{1}^{i}, \ldots a_{d_{i}}^{i}\right\}$ is a basis of $W_{i}^{\varphi}$ we can choose elements $\left\{{ }_{m} a_{1}^{i}, \ldots{ }_{m} a_{d_{i}}^{i}\right\} \subset k\left\langle A_{m}^{i}\right\rangle$ $(m \geq 1)$ such that $\tilde{\psi}_{i}^{m}\left(a_{j}^{i}\right)={ }_{m} a_{j}^{i}+\varphi\left(k\left\langle B_{M_{m}}^{i}\right\rangle\right)$. In particular, since the homomorphisms $\tilde{\psi}_{i}^{m}$ are compatible with the bonding homomorphisms of $Y_{\bullet}^{i}$, we see that there are elements ${ }_{m+1} b_{j}^{i} \in k\left\langle B_{M_{m}}^{i}\right\rangle$ satisfying ${ }_{m+1} a_{j}^{i}-{ }_{m} a_{j}^{i}=\varphi\left({ }_{m+1} b_{j}^{i}\right)$. Moreover, let $\left\{a_{1}, \ldots a_{d}\right\}$ be a basis of $W_{0}^{\varphi}, \sigma: k\langle A\rangle / \varphi(k\langle B\rangle) \hookrightarrow k\langle A\rangle$ a splitting of the natural projection, and elements ${ }_{1} b_{j}^{i} \in k\langle B\rangle$ such that $\varphi\left({ }_{1} b_{j}^{i}\right)={ }_{1} a_{j}^{i}-\sigma\left({ }_{1} a_{j}^{i}+\varphi(k\langle B\rangle)\right)$.

If $\rho$ is the finite presentation of MSM constructed in the proof of (9.1), there is a morphism $\tau: \rho \rightarrow \varphi$ in $\operatorname{pair}\left(\mathbf{M}_{k}\left(\bar{T}_{n}\right)\right)$ given by $\tau_{0}\left(w_{i}\right)=\sigma\left(a_{i}\right), \tau_{0}\left({ }_{m} w_{j}^{i}\right)={ }_{m} a_{j}^{i}$, and $\tau_{1}\left({ }_{m} w_{j}^{i}\right)={ }_{m} b_{j}^{i}$. This morphism induces a $\mathbf{M}_{k}\left(\bar{T}_{n}\right)$-module morphism $\tau: \mathbb{M S} \mathcal{M} \rightarrow$ $\mathcal{M}$. Now we are going to construct a retraction of $\tau$.

By (9.4) $Z_{m}^{i}$ is always finite-dimensional and $W_{i}^{\varphi}=\cap_{m \geq 1} Z_{m}^{i}$, hence there exists $N \geq 1$ such that $W_{i}^{\varphi}=Z_{N}^{i}$ for every $1 \leq i \leq n$. Let $\underline{V}$ be the $n$-subspace given by $V_{0}=k\langle A\rangle / \varphi(k\langle B\rangle)$ and $V_{i}=\left[k\left\langle A_{N}^{i}\right\rangle+\varphi(k\langle B\rangle)\right] / \varphi(k\langle B\rangle)$. Clearly $\mathbb{S} \mathcal{M}=$ $\underline{V}^{\text {rig }} \subset \underline{V}$, hence by (8.4) there is a retraction $r: \underline{V} \rightarrow \mathbb{S} \mathcal{M}$. By Yoneda's lemma $\operatorname{Hom}_{\mathcal{R}}\left(k\langle A\rangle_{\alpha}, \mathbb{M} \underline{\underline{V}}\right)=\mathbb{M} \underline{\underline{V}}\left(k\langle A\rangle_{\alpha}\right)$. The natural projection $k\langle A\rangle \rightarrow V_{0}$ give rise to a $\mathbf{M}_{k}\left(\bar{T}_{n}\right)$-module morphism $v_{0}: k\langle A\rangle_{\alpha} \rightarrow \mathbb{M} \underline{\underline{V}}$ such that $v_{0} \varphi=0$. Moreover, since $\mathcal{M}=\operatorname{Coker} \varphi$ then $\operatorname{Hom}_{\mathcal{R}}(\mathcal{N}, \mathbb{M} \underline{V})=\operatorname{Ker} \operatorname{Hom}_{\mathcal{R}}(\varphi, \mathbb{M} \underline{V})$, in particular $v_{0}$ determines a $\mathbf{M}_{k}\left(\bar{T}_{n}\right)$-module morphism $v: \mathcal{N} \rightarrow \mathbb{M} \underline{\underline{V}}$. One readily checks that the composition $(\mathbb{M} r) v_{0} \tau_{0}$ coincides with the natural projection $p: k\langle C\rangle_{\gamma} \rightarrow \mathbb{M S M}=$ Coker $\rho$ defined in the proof of $(9.1)$, hence $(\mathbb{M} r) v \tau=1$ is the identity on $\mathbb{M S} \mathcal{N}$, and $(\mathbb{M} r) v$ is the desired retraction of $\tau$. Now if we take $\mathcal{N}$ to be the cokernel of $\tau$ the proposition follows since $\mathcal{M}=\mathcal{N} \oplus \mathbb{M} \mathbb{S} \mathcal{M}$, by (9.8) $\mathbb{S} \mathcal{M}=\mathbb{S} \mathcal{N} \oplus \mathbb{S M S M} \simeq \mathbb{S} \mathcal{N} \oplus \mathbb{S} \mathcal{M}$ and hence $\mathbb{S N}=0$. Here we use that the monoid $\operatorname{Iso}\left(\mathbf{s u b}_{n}^{\text {fin }}\right)$ is free and hence cancelative, compare Section 8.

In the next proposition we use the change of coefficients $\mathbb{F}_{*}^{i}$ associated to the additive functors $\mathbb{F}^{i}: \mathbf{M}_{k}\left(\bar{T}_{1}\right) \rightarrow \mathbf{M}_{k}\left(\bar{T}_{n}\right)$ in Remark 6.5.

Proposition 9.10. Given a f. p. $\mathbf{M}_{k}\left(\bar{T}_{n}\right)$-module $\mathcal{N}, \mathbb{S} \mathcal{M}=0$ if and only if there exist f. p. $\mathbf{M}_{k}\left(\bar{T}_{1}\right)$-modules $\mathcal{M}_{i}(1 \leq i \leq n)$ with $\mathcal{M} \simeq \mathbb{F}_{*}^{1} \mathcal{M}_{1} \oplus \cdots \oplus \mathbb{F}_{*}^{n} \mathcal{M}_{n}$.

Proof. It is easy to see that $\mathbb{S F}_{*}^{i}=0(1 \leq i \leq n)$, and $\mathbb{S}$ is additive, so the implication $\Leftarrow$ follows. Now suppose that $\mathcal{M}=\operatorname{Coker}\left[\varphi: k\langle B\rangle_{\beta} \rightarrow k\langle A\rangle_{\alpha}\right]$ and $\mathbb{S} \mathcal{M}=0$. Since finite-dimensional vector spaces are artinian, by (9.4) there exists $m \geq 1$ big enough such that for every $1 \leq i \leq n$,

$$
\frac{\left[k\left\langle A_{m}^{i}\right\rangle+\varphi(k\langle B\rangle)\right] \cap\left[\sum_{j \neq i} k\left\langle A_{m}^{j}\right\rangle+\varphi(k\langle B\rangle)\right]}{\varphi(k\langle B\rangle)}=0,
$$

it is, the following equality holds (the isomorphism on the right always holds)

$$
\frac{\sum_{i=1}^{n} k\left\langle A_{m}^{i}\right\rangle+\varphi(k\langle B\rangle)}{\varphi(k\langle B\rangle)}=\bigoplus_{i=1}^{n} \frac{k\left\langle A_{m}^{i}\right\rangle+\varphi(k\langle B\rangle)}{\varphi(k\langle B\rangle)} \simeq \bigoplus_{i=1}^{n} \frac{k\left\langle A_{m}^{i}\right\rangle}{k\left\langle A_{m}^{i}\right\rangle \cap \varphi(k\langle B\rangle)} .
$$

This is equivalent to state that

$$
\left[\bigoplus_{i=1}^{n} k\left\langle A_{m}^{i}\right\rangle\right] \cap \varphi(k\langle B\rangle)=\bigoplus_{i=1}^{n}\left[k\left\langle A_{m}^{i}\right\rangle \cap \varphi(k\langle B\rangle)\right] .
$$


By the characterization of controlled homomorphisms in Subsection 3.1 there exists $M \geq 1$ with $\varphi\left(B_{M}^{i}\right) \subset k\left\langle A_{m}^{i}\right\rangle(1 \leq i \leq n)$. Let $K$ be the kernel of the vector space homomorphism underlying to $\varphi$, it is $K=\varphi^{-1}(0)$. There is a finite set $\left\{b_{1}, \ldots b_{d}\right\} \subset k\langle B\rangle$ which projects to a basis of

$$
\frac{K+\left(\bigoplus_{i=0}^{n} k\left\langle B_{M}^{i}\right\rangle\right)}{\bigoplus_{i=0}^{n} k\left\langle B_{M}^{i}\right\rangle} \simeq \frac{K}{K \cap\left(\bigoplus_{i=0}^{n} k\left\langle B_{M}^{i}\right\rangle\right)},
$$

since this vector space is contained in

$$
\frac{k\langle B\rangle}{\bigoplus_{i=0}^{n} k\left\langle B_{M}^{i}\right\rangle} \simeq k\left\langle_{M-1} B\right\rangle
$$

and ${ }_{M-1} B$ is finite.

There is also a finite set $\left\{a_{1}^{i}, \ldots a_{d_{i}}^{i}\right\} \subset k\langle B\rangle$ which projects to a basis of

$$
\frac{\varphi^{-1}\left(k\left\langle A_{m}^{i}\right\rangle \cap \varphi(k\langle B\rangle)\right)}{k\left\langle B_{M}^{i}\right\rangle+K},
$$

because $\varphi$ induces an isomorphism

$$
\frac{\varphi^{-1}\left(k\left\langle A_{m}^{i}\right\rangle \cap \varphi(k\langle B\rangle)\right)}{k\left\langle B_{M}^{i}\right\rangle+K} \simeq \frac{k\left\langle A_{m}^{i}\right\rangle \cap \varphi(k\langle B\rangle)}{\varphi\left(k\left\langle B_{M}^{i}\right\rangle\right)},
$$

and always

$$
k\left\langle A_{m}^{i}\right\rangle \cap\left(\sum_{j \neq i} k\left\langle A_{m}^{j}\right\rangle\right)=0
$$

so $k\left\langle A_{m}^{i}\right\rangle \cap\left[\sum_{j=1}^{n} \varphi\left(k\left\langle B_{M}^{j}\right\rangle\right)\right]=\varphi\left(k\left\langle B_{M}^{i}\right\rangle\right)$, and hence

$\frac{k\left\langle A_{m}^{i}\right\rangle \cap \varphi(k\langle B\rangle)}{\varphi\left(k\left\langle B_{M}^{i}\right\rangle\right)}=\frac{k\left\langle A_{m}^{i}\right\rangle \cap \varphi(k\langle B\rangle)}{k\left\langle A_{m}^{i}\right\rangle \cap\left[\sum_{j=1}^{n} \varphi\left(k\left\langle B_{M}^{j}\right\rangle\right)\right]} \subset \frac{\varphi(k\langle B\rangle)}{\sum_{i=1}^{n} \varphi\left(k\left\langle B_{M}^{i}\right\rangle\right)} \simeq \varphi\left(k\left\langle_{M-1} B\right\rangle\right)$.

By (b) we have that

$$
\varphi^{-1}\left(k\left\langle A_{m}^{i}\right\rangle \cap \varphi(k\langle B\rangle)\right) \cap\left[\sum_{j \neq i} \varphi^{-1}\left(k\left\langle A_{m}^{j}\right\rangle \cap \varphi(k\langle B\rangle)\right)\right]=K,
$$

therefore the set $\left[\sqcup_{i=1}^{n}\left(B_{M}^{i} \sqcup\left\{a_{j}^{i}\right\}_{j=1}^{d_{i}}\right)\right] \sqcup\left\{b_{i}\right\}_{i=1}^{d}$ is linearly independent in $k\langle B\rangle$. Moreover, it is a basis of $\sum_{i=1}^{n} \varphi^{-1}\left(k\left\langle A_{m}^{i}\right\rangle \cap \varphi(k\langle B\rangle)\right)$, so in order to complete it to a basis $\underline{B}$ of $k\langle B\rangle$ we only need to add a finite set $\left\{b_{1}^{\prime}, \ldots b_{d^{\prime}}^{\prime}\right\} \subset k\langle B\rangle$ which projects to a basis of the following vector space

$$
\frac{k\langle B\rangle}{\sum_{i=1}^{n} \varphi^{-1}\left(k\left\langle A_{m}^{i}\right\rangle \cap \varphi(k\langle B\rangle)\right)} .
$$

This vector space is isomorphic to

$$
\frac{\varphi(k\langle B\rangle)}{\bigoplus_{i=1}^{n} k\left\langle A_{m}^{i}\right\rangle \cap \varphi(k\langle B\rangle)} \subset \frac{k\langle A\rangle}{\bigoplus_{i=1}^{n} k\left\langle A_{m}^{i}\right\rangle} \simeq k\left\langle_{m-1} A\right\rangle
$$

and hence finite-dimensional. The inclusion (c) follows from (a). Let $\left\{a_{1}, \ldots a_{e}\right\} \subset$ $k\langle A\rangle$ be a basis of

$$
\frac{k\langle A\rangle}{\varphi(k\langle B\rangle)+\left(\bigoplus_{i=1}^{n} k\left\langle A_{m}^{i}\right\rangle\right)}
$$


By (c) $\underline{A}=\left(\sqcup_{i=1}^{n} A_{m}^{i}\right) \sqcup\left\{\varphi\left(b_{i}^{\prime}\right)\right\}_{i=1}^{d^{\prime}} \sqcup\left\{a_{i}\right\}_{i=1}^{e}$ is a basis of $k\langle A\rangle$. Let $\underline{\alpha}: \underline{A} \rightarrow T_{n}^{0}$, $\underline{\beta}: \underline{B} \rightarrow T_{n}^{0}$ be the height functions defined as $\alpha$ and $\beta$ over $\sqcup_{i=1}^{n} A_{m}^{i}$ and $\sqcup_{i=1}^{n} B_{M}^{i}$ respectively, and constant $v_{0}$ on the other elements. The identities $k\langle A\rangle=k\langle\underline{A}\rangle$ and $k\langle B\rangle=k\langle\underline{B}\rangle$ induce controlled isomorphisms $\phi_{1}: k\langle A\rangle_{\alpha} \simeq k\langle\underline{A}\rangle_{\underline{\alpha}}$ and $\phi_{2}: k\langle B\rangle_{\beta} \simeq$ $k\langle\underline{B}\rangle_{\beta}$, so if we define $\psi=\phi_{1} \varphi \phi_{2}^{-1}$ then $\mathcal{M} \simeq$ Coker $\psi$. But if we define the sets ${ }^{1} \underline{A}=A_{m}^{1} \sqcup\left\{\varphi\left(b_{i}^{\prime}\right)\right\}_{i=1}^{d^{\prime}} \sqcup\left\{a_{i}\right\}_{i=1}^{e},{ }^{i} \underline{A}=A_{m}^{i}(1<i \leq n),{ }^{1} \underline{B}=B_{M}^{1} \sqcup\left\{a_{j}^{1}\right\}_{j=1}^{d_{1}} \sqcup$ $\left\{b_{i}\right\}_{i=1}^{d} \sqcup\left\{b_{i}^{\prime}\right\}_{i=1}^{d^{\prime}},{ }^{i} \underline{B}=B_{M}^{i} \sqcup\left\{a_{j}^{i}\right\}_{j=1}^{d_{i}}(1<i \leq n)$, and the height functions ${ }^{i} \underline{\alpha}$ and ${ }^{i} \underline{\beta}$ as the restriction of $\underline{\alpha}$ and $\underline{\beta}$ to ${ }^{i} \underline{A}$ and ${ }^{i} \underline{B}$ respectively $(1 \leq i \leq n)$, then we observe that $(1 \leq i \leq n)$

$$
\begin{gathered}
{ }^{i} \underline{\alpha}\left({ }^{i} \underline{A}\right),{ }^{i} \underline{\beta}\left({ }^{i} \underline{B}\right) \subset\left\{v_{0}\right\} \cup\left\{v_{m}^{i}\right\}_{m \geq 1} \subset T_{n}^{0}, \\
\psi\left({ }^{i} \underline{A}\right) \subset k\left\langle{ }^{i} \underline{B}\right\rangle,
\end{gathered}
$$

and the proposition follows.

\section{Classification of finitely PResented $k(\boldsymbol{n})$-Modules}

In this final section we complete the proofs of Theorems 1.1 and 1.2. For this, the crucial result is the next theorem where we compute the monoid $\operatorname{Iso}(\mathbf{f} \mathbf{p}(k(\mathbf{n})))$ in terms of the free abelian monoid Iso $\left(\mathbf{s u b}_{n}^{\mathrm{fr}}\right)$.

Theorem 10.1. The following monoid morphism is an isomorphism for every $n \in \mathbb{N}$ :

$$
\left(\Phi_{n}, \operatorname{Iso}(\mathbb{S})\right): \operatorname{Iso}(\mathbf{f p}(k(\mathbf{n}))) \stackrel{\simeq}{\longrightarrow} \mathbb{N}_{\infty, n} \times \prod_{i=1}^{n} \mathbb{N}_{\infty} \times \prod_{i=1}^{n} \mathbb{N}_{\infty} \times \operatorname{Iso}\left(\mathbf{s u b}_{n}^{\mathrm{fr}}\right) .
$$

This theorem follows from the strongest results previously proven in this paper. More precisely, the surjectivity of $\left(\Phi_{n}, \mathrm{Iso}(\mathbb{S})\right)$ is a consequence of $(6.6),(7.3),(9.2)$, (9.7), (9.8) and (9.10). Furthermore, this morphism is injective by the next theorem. In order to state it we introduce the following notation. Given $d \in \mathbb{N}_{\infty, n}$ the $k(\mathbf{n})$-module $\mathcal{A}_{d}$ is $\mathbb{F}_{*}^{1} \mathcal{A}_{d}$ provided $d \in \mathbb{N}_{0}$ and $\oplus_{i \in S} \mathbb{F}_{*}^{i} \mathcal{R}$ if $d=\infty_{S}$ for some $\emptyset \neq S \subset\{1, \ldots n\}$.

Theorem 10.2. Any f. p. $k(\mathbf{n})$-module $\mathcal{M}$ decomposes in the following way

$$
\mathcal{M} \simeq \mathcal{A}_{\lambda_{\mathcal{M}}} \oplus\left(\bigoplus_{i=1}^{n} \mathbb{F}_{*}^{i} \mathcal{B}_{\mu_{\mathcal{M}}^{i}}\right) \oplus\left(\bigoplus_{i=1}^{n} \mathbb{F}_{*}^{i} \mathcal{C}_{\nu_{\mathcal{M}}^{i}}\right) \oplus \mathbb{M} \mathcal{S} \mathcal{M}
$$

This theorem follows from (6.6), (7.2), (9.9), (9.10), and the following

Lemma 10.3. Given two f. p. $k(\mathbf{1})$-modules $\mathcal{M}$ and $\mathcal{N}$ there exists a $k(\mathbf{n})$-module isomorphism $\mathbb{F}_{*}^{i} \mathcal{N} \simeq \mathbb{F}_{*}^{j} \mathcal{N}(1 \leq i, j \leq n)$ if and only if one of the following conditions is satisfied:

- $i=j$ and $\mathcal{M} \simeq \mathcal{N}$,

- $\mathcal{M} \simeq \mathcal{N} \simeq \mathcal{A}_{n}$ for some $n \in \mathbb{N}_{0}$.

Proof. The implication $\Rightarrow$ follows from (6.6), (7.3) and (7.4). On the other hand if $\mathcal{M} \simeq \mathcal{N}$ then obviously $\mathbb{F}_{*}^{i} \mathcal{N} \simeq \mathbb{F}_{*}^{i} \mathcal{N}$. Moreover, $\mathbb{F}_{*}^{i} \mathcal{A}_{n}$ and $\mathbb{F}_{*}^{j} \mathcal{A}_{n}$ are isomorphic $\left(n \in \mathbb{N}_{0}\right)$ because both modules are isomorphic to a free $\bar{T}_{n}$-controlled $k$-vector space whose basis is a set with $n$ elements, compare (7.7).

As a consequence of Proposition 8.5 and Theorem 10.1 we obtain the next 
Corollary 10.4. The algebra $k(\mathbf{n})$ has the same representation type as the $n$ subspace quiver.

Now Theorem 1.1 follows from (3.10), (8.6) and (10.4).

We obtain from (6.6), (7.3), (10.1) and (10.2) the following presentation of the $\operatorname{monoid} \operatorname{Iso}(\mathbf{f p}(k(\mathbf{n})))$.

Corollary 10.5 (Classification of f. p. $k(\mathbf{n})$-modules). Let $\left\{\underline{V}^{(n, j)}\right\}_{j \in J_{n}}$ be the set of indecomposable rigid n-subspaces. There is a solution to the decomposition problem in the category of $f . p . k(\mathbf{n})$-modules given by the following $1+5 n+$ card $J_{n}$ elementary modules $\left(1 \leq i \leq n, j \in J_{n}\right)$

$$
\mathbb{F}_{*}^{1} \mathcal{A}, \mathbb{F}_{*}^{i} \mathcal{R}, \mathbb{F}_{*}^{i} \mathcal{B}, \mathbb{F}_{*}^{i} \mathcal{B}_{\infty}, \mathbb{F}_{*}^{i} \mathcal{C}, \mathbb{F}_{*}^{i} \mathcal{C}_{\infty}, \mathbb{M} \underline{V}^{(n, j)},
$$

and 6 n elementary isomorphisms $(1 \leq i \leq n)$

$$
\begin{gathered}
\mathbb{F}_{*}^{1} \mathcal{A} \oplus \mathbb{F}_{*}^{i} \mathcal{R} \simeq \mathbb{F}_{*}^{i} \mathcal{R}, \mathbb{F}_{*}^{i} \mathcal{R} \oplus \mathbb{F}_{*}^{i} \mathcal{R} \simeq \mathbb{F}_{*}^{i} \mathcal{R}, \mathbb{F}_{*}^{i} \mathcal{B} \oplus \mathbb{F}_{*}^{i} \mathcal{B}_{\infty} \simeq \mathbb{F}_{*}^{i} \mathcal{B}_{\infty}, \\
\mathbb{F}_{*}^{i} \mathcal{B}_{\infty} \oplus \mathbb{F}_{*}^{i} \mathcal{B}_{\infty} \simeq \mathbb{F}_{*}^{i} \mathcal{B}_{\infty}, \mathbb{F}_{*}^{i} \mathcal{C} \oplus \mathbb{F}_{*}^{i} \mathcal{C}_{\infty} \simeq \mathbb{F}_{*}^{i} \mathcal{C}_{\infty}, \mathbb{F}_{*}^{i} \mathcal{C}_{\infty} \oplus \mathbb{F}_{*}^{i} \mathcal{C}_{\infty} \simeq \mathbb{F}_{*}^{i} \mathcal{C}_{\infty} .
\end{gathered}
$$

This classification theorem together with (8.7) and (8.8) complete the proof of Theorem 1.2.

\section{Appendix A. Some COMputations of $\operatorname{Ext}_{k(\boldsymbol{n})}^{1}$ Groups}

The aim of this appendix is to provide with tools and techniques to compute the $\operatorname{Ext}_{k(\mathbf{n})}^{1}$ group of any pair of f. p. $k(\mathbf{n})$-modules. This group is in fact a $k$ vector space, so it is determined by its dimension. Higher $\operatorname{Ext}_{k(\mathbf{n})}^{*} \operatorname{groups}_{\text {vanish }}$ over f. p. $k(\mathbf{n})$-modules by (7.16). Since the functor $\operatorname{Ext}_{k(\boldsymbol{n})}^{1}$ is biadditive we just have to compute it over pairs of elementary f. p. $k(\mathbf{n})$-modules, see (10.5). We shall not make all these computations here for an arbitrary $n$, but just for $n=1$, $k(\mathbf{1})=\operatorname{RCFM}(k)$. In addition we show for any $n \in \mathbb{N}$ that the $\operatorname{Ext}_{k(\boldsymbol{n})}^{1}$ of pairs of f. p. $k(\boldsymbol{n})$-modules coming from finite-dimensional $n$-subspaces via the functor $\mathbb{M}$ in (9.A) coincide with their $\operatorname{Ext}_{k Q_{n}}^{1}$ as modules over the path algebra. This last vector space is much easier to compute, since one can use the integral bilinear form of the quiver $Q_{n}$, see [16].

Let $\mathcal{R}$ be the $k$-algebra $\operatorname{RCFM}(k)$ as in Section 7 . Given two elementary $\mathcal{R}$ modules $\mathcal{R} / Y \mathcal{R}(Y \neq 0)$ and $\mathcal{R} / Z \mathcal{R}$, see (7.1), one can check by using (7.9) and basic homological algebra that there is an isomorphism of $k$-vector spaces

$$
\operatorname{Ext}_{\mathcal{R}}^{1}(\mathcal{R} / \mathrm{Y} \mathcal{R}, \mathcal{R} / \mathrm{Z} \mathcal{R}) \simeq \frac{\mathcal{R}}{\mathcal{R Y}+\mathrm{Z} \mathcal{R}}
$$

This formula also holds for $\mathbf{Z}=0$, moreover, in this case it is a left- $\mathcal{R}$-module isomorphism.

Lemma A.1. We have the following identities

(1) $\mathrm{A} \mathcal{R}=\left\{\mathrm{R} \in \mathcal{R} ; \mathrm{r}_{0 j}=0\right.$ for all $\left.j \in \mathbb{N}_{0}\right\}$,

(2) $(\mathrm{I}-\mathrm{A}) \mathcal{R}=\left\{\mathrm{R} \in \mathcal{R} ; \sum_{i \in \mathbb{N}_{0}} \mathrm{r}_{i j}=0\right.$ for all $\left.j \in \mathbb{N}_{0}\right\}$,

(3) $\left(\mathrm{I}-\mathrm{A}^{t}\right) \mathcal{R}=\left\{\mathrm{R} \in \mathcal{R} ;\right.$ given any $i \in \mathbb{N}_{0}, \sum_{n \geq i} \mathrm{r}_{n j}=0$ for almost all $\left.j \in \mathbb{N}_{0}\right\}$,

(4) $\left(\mathrm{I}-\mathrm{B}^{t}\right) \mathcal{R}=\left\{\mathrm{R} \in \mathcal{R} ;\right.$ given $m \in \mathbb{N}_{0}$ and $i \leq m, \sum_{n \geq m} \mathrm{r}_{i+\frac{n(n+1)}{2}, j}=0$ for almost all $\left.j \in \mathbb{N}_{0}\right\}$. 
Proof. One can check that the right-hand-side sets of the statement are ideals, hence in order to establish the inclusions $\subset$ it is enough to prove that the matrix defining each left-hand-side ideal belongs to the corresponding right-hand-side set. This can be checked by a maybe tedious but straightforward computation. Suppose now that $\mathrm{R}$ is a matrix in the right-hand-side set of (1), (2), (3), or (4), then one can check that the matrix $\mathrm{C}^{1}, \mathrm{C}^{2}, \mathrm{C}^{3}$ or $\mathrm{C}^{4}$ defined as $c_{0 j}^{1}=0, \mathrm{c}_{i+1, j}^{1}=\mathrm{r}_{i j}, \mathrm{c}_{i j}^{2}=\sum_{n=0}^{i} \mathrm{r}_{n j}$, $\mathrm{c}_{i j}^{3}=\sum_{n \geq i} \mathrm{r}_{n j}(i, j \in \mathbb{N}), \mathrm{c}_{i+\frac{m(m+1)}{2}, j}^{4}=\sum_{n \geq m} \mathrm{r}_{i+\frac{n(n+1)}{2}, j}\left(i, j \in \mathbb{N}_{0}, m \geq i\right)$, belongs to $\mathcal{R}$ and satisfies $A C^{1}=R,(I-A) C^{2}=R,\left(I-A^{t}\right) C^{3}=R$ or $\left(I-B^{t}\right) C^{4}=R$, provided we are in case (1), (2), (3) or (4). Hence we are done.

By using Lemma A.1 and the involution of the $k$-vector space $\mathcal{R}$ given by transposition of matrices, one readily checks that

Lemma A.2. We have the following equalities

(1) $\mathcal{R}(\mathrm{I}-\mathrm{A})=\left\{\mathrm{R} \in \mathcal{R} ;\right.$ given any $j \in \mathbb{N}_{0}, \sum_{n \geq j} \mathrm{r}_{\text {in }}=0$ for almost all $\left.i \in \mathbb{N}_{0}\right\}$,

(2) $\mathcal{R}\left(\mathrm{I}-\mathrm{A}^{t}\right)=\left\{\mathrm{R} \in \mathcal{R} ; \sum_{j \in \mathbb{N}_{0}} \mathrm{r}_{i j}=0\right.$ for all $\left.i \in \mathbb{N}_{0}\right\}$,

(3) $\mathcal{R}(\mathrm{I}-\mathrm{B})=\left\{\mathrm{R} \in \mathcal{R} ;\right.$ given $m \in \mathbb{N}_{0}$ and $j \leq m, \sum_{n \geq m} \mathrm{r}_{i, j+\frac{n(n+1)}{2}}=0$ for almost all $\left.i \in \mathbb{N}_{0}\right\}$.

Proposition A.3. We have that

(1) $\operatorname{dim} \operatorname{Ext}_{\mathcal{R}}^{1}(\mathcal{B}, \mathcal{R})=2^{\aleph_{0}}$,

(2) $\operatorname{dim} \operatorname{Ext}_{\mathcal{R}}^{1}(\mathcal{C}, \mathcal{R})=2^{\aleph_{0}}$,

(3) $\operatorname{dim} \operatorname{Ext}_{\mathcal{R}}^{1}\left(\mathcal{B}_{\infty}, \mathcal{R}\right)=2^{\aleph_{0}}$,

(4) $\operatorname{dim} \operatorname{Ext}_{\mathcal{R}}^{1}\left(\mathcal{C}_{\infty}, \mathcal{R}\right)=2^{\aleph_{0}}$,

(5) $\operatorname{dim} \operatorname{Ext}_{\mathcal{R}}^{1}(\mathcal{B}, \mathcal{A})=0$,

(6) $\operatorname{dim} \operatorname{Ext}_{\mathcal{R}}^{1}(\mathcal{C}, \mathcal{A})=1$,

(7) $\operatorname{dim} \operatorname{Ext}_{\mathcal{R}}^{1}\left(\mathcal{B}_{\infty}, \mathcal{A}\right)=0$

(8) $\operatorname{dim} \operatorname{Ext}_{\mathcal{R}}^{1}\left(\mathcal{C}_{\infty}, \mathcal{A}\right)=\aleph_{0}$.

Proof. One can check by using (A.2) (1), (A.1) (1) and (A.2) (2), and (A.1) (1) and (A.2) (3), that there are $k$-vector space isomorphisms

$$
\begin{gathered}
\frac{\mathcal{R}}{\mathcal{R}(\mathrm{I}-\mathrm{A})} \simeq \frac{\prod_{i \in \mathbb{N}_{0}} k}{\bigoplus_{i \in \mathbb{N}_{0}} k}, \mathrm{R}+\mathcal{R}(\mathrm{I}-\mathrm{A}) \mapsto\left(\sum_{j \in \mathbb{N}_{0}} \mathrm{r}_{i j}\right)_{i \in \mathbb{N}_{0}}+\bigoplus_{i \in \mathbb{N}_{0}} k, \\
\frac{\mathcal{R}}{\mathcal{R}\left(\mathrm{I}-\mathrm{A}^{t}\right)+\mathrm{A} \mathcal{R}} \simeq k, \mathrm{R}+\left(\mathcal{R}\left(\mathrm{I}-\mathrm{A}^{t}\right)+\mathrm{A} \mathcal{R}\right) \mapsto \sum_{j \in \mathbb{N}_{0}} \mathrm{r}_{0 j}, \\
\frac{\mathcal{R}}{\mathcal{R}\left(\mathrm{I}-\mathrm{B}^{t}\right)+\mathrm{A} \mathcal{R}} \simeq \bigoplus_{j \in \mathbb{N}_{0}} k, \mathrm{R}+\left(\mathcal{R}\left(\mathrm{I}-\mathrm{B}^{t}\right)+\mathrm{A} \mathcal{R}\right) \mapsto\left(\sum_{n \geq j} \mathrm{r}_{0, j+\frac{n(n+1)}{2}}\right)_{j \in \mathbb{N}_{0}},
\end{gathered}
$$

hence (1), (6) and (8) follow from (A.A).

For any pair of elementary f. p. R-modules the inequality $\operatorname{dim} \operatorname{Ext}_{\mathcal{R}}^{1} \leq 2^{\aleph_{0}}$ follows from (A.A) and (3.8). Now (2) is a consequence of (1) and (7.10) (1), (3) follows from (1) and the fact that $\mathcal{B}$ is a direct summand of $\mathcal{B}_{\infty}$, see (7.1), and (4) is a consequence of (3) and (7.10) (2).

Given any matrix $\mathbf{R} \in \mathcal{R}$, if $\mathbf{R}^{1}, \mathbf{R}^{2} \in \mathcal{R}$ are the matrices defined by $r_{0 j}^{1}=r_{0 j}$, $\mathrm{r}_{i j}^{2}=\mathrm{r}_{i j}(i>0)$, and $\mathrm{r}_{i j}^{n}=0$ otherwise, then $\mathrm{R}=\mathrm{R}^{1}+\mathrm{R}^{2}, \mathrm{R}^{1} \in \mathcal{R}(\mathrm{I}-\mathrm{B})$ and $\mathrm{R}^{2} \in \mathrm{A} \mathcal{R}$, by (A.1) (1) and (A.2) (3), hence $\mathcal{R}=\mathcal{R}(\mathrm{I}-\mathrm{B})+\mathrm{A} \mathcal{R}$ and (7) follows 
by (A.A). Moreover, since $\mathcal{B}$ is a direct summand of $\mathcal{B}_{\infty}$ by (7.1) then (5) also follows.

By (7.1), (7.12) and (7.17) the $\operatorname{Ext}_{\mathcal{R}}^{1}$ group of any other pair of elementary f. p. $\mathcal{R}$-modules is zero, hence the first extension groups are now completely computed for f. p. R-modules.

Proposition A.4. For any pair of finite-dimensional n-subspaces there is a natural isomorphism

$$
\operatorname{Ext}_{k Q_{n}}^{1}(\underline{V}, \underline{W}) \simeq \operatorname{Ext}_{k(n)}^{1}(\mathbb{M} \underline{V}, \mathbb{M} \underline{W}) .
$$

Proof. Projective representations of $Q_{n}$ are (arbitrary) direct sums of the following $n+1$ indecomposable $n$-subspaces,

$$
\mathbb{F}^{1}(0 \rightarrow k), \mathbb{F}^{i}(k \rightarrow k) \quad(1 \leq i \leq n) .
$$

Since $\mathbb{M}$ is an exact full inclusion of categories and any finite-dimensional representation of $Q_{n}$ admits a length-one projective resolution by finite-dimensional projective representations it is enough to check that

(1) $\operatorname{Ext}_{k(\mathbf{n})}^{1}\left(\mathbb{M F}^{1}(0 \rightarrow k), \mathbb{M F}^{1}(0 \rightarrow k)\right)=0$,

(2) $\operatorname{Ext}_{k(\boldsymbol{n})}^{1}\left(\mathbb{M F}^{1}(0 \rightarrow k), \mathbb{M F}^{i}(k \rightarrow k)\right)=0(1 \leq i \leq n)$,

(3) $\operatorname{Ext}_{k(\mathbf{n})}^{1}\left(\mathbb{M F}^{i}(k \rightarrow k), \mathbb{M F}^{1}(0 \rightarrow k)\right)=0(1 \leq i \leq n)$,

(4) $\operatorname{Ext}_{k(\mathbf{n})}^{1}\left(\mathbb{M F}^{i}(k \rightarrow k), \mathbb{M F}^{j}(k \rightarrow k)\right)=0(1 \leq i, j \leq n)$.

The resolution constructed in the proof of $(9.1)$ shows that $\mathbb{M F}^{1}(0 \rightarrow k)$ is a projective $k(\mathbf{n})$-module isomorphic to a 1-dimensional $\bar{T}_{n}$-controlled $k$-vector space, hence (1) and (2) hold. Moreover, one can easily check (3) and (4) by using the definition of $\mathbb{M}$ in (9.A) and the resolutions of $\mathbb{M F}^{i}(k \rightarrow k)=0(1 \leq i \leq n)$ in the proof of (9.1).

\section{REFERENCES}

1. R. Ayala, M. Cárdenas, F. Muro, and A. Quintero, An elementary approach to projective dimension in proper homotopy theory, To appear in Comm. in Alg.

2. H.-J. Baues and A. Quintero, Infinite Homotopy Theory, K-Monographs in Mathematics, no. 6, Kluwer, 2001.

3. F. Borceux, Handbook of categorical algebra 1, Encyclopedia of Math. and its Applications, no. 50, Cambridge University Press, 1994.

4. - Handbook of categorical algebra 2, Encyclopedia of Math. and its Applications, no. 51, Cambridge University Press, 1994.

5. G. Carlsson and E. K. Pedersen, Controlled algebra and the Novikov conjectures for K- and L-theory, Topology 34 (1995), 731-758.

6. J.-M. Cordier and T. Porter, Shape Theory, Ellis Horwood, 1989.

7. F. T. Farrell and J. B. Wagoner, Infinite matrices in algebraic K-theory and topology, Comment. Math. Helv. 47 (1972), 474-501.

8. P. Gabriel, Unzerlegbare Darstellungen I, Manuscripta Math. 6 (1972), 71-103.

9. N. Higson, E. K. Pedersen, and J. Roe, $C^{*}$-algebras and controlled topology, $K$-theory 11 (1997), 209-239.

10. P. J. Hilton and U. Stammbach, A Course in Homological Algebra, Springer-Verlag, New York, 1971.

11. S. Mardešic and J. Segal, Shape Theory, Math. Library, no. 26, North-Holland, 1982.

12. B. Mitchell, Rings with several objects, Advances in Mathematics 8 (1972), 1-161.

13. F. Muro, On the proper homotopy classification of locally compact $A_{n}^{2}$-polyhedra, In preparation.

14. L. A. Nazarova, Representations of quivers of inifinite type, Math. USSR Izvestija 7 (1973), no. $4,749-792$. 
15. F. Quinn, Geometric Algebra. Algebraic and Geometric Topology, Lecture Notes in Mathematics, no. 1126, Springer-Verlag, Berlin Heidelberg, 1985.

16. C. M. Ringel, Tame algebras and integral quadratic forms, Lecture Notes in Mathematics, no. 1099, Springer-Verlag, Berlin, 1984.

17. J. J. Simón, Finitely generated projective modules over row and column finite matrix rings, J. Algebra 208 (1998), 165-184.

18. J. B. Wagoner, Delooping classifying spaces in algebraic K-theory, Topology 11 (1972), 349370.

Departamento de Geometría y Topología. Universidad de Sevilla.

E-mail address: fmuro@us.es 